\title{
Time Sensitive Analysis of Antagonistic Stochastic Processes and Applications to Finance and Queueing
}

\author{
Jewgeni H. Dshalalow ${ }^{1, *}$, Kizza Nandyose ${ }^{2}$, Ryan T. White ${ }^{1}$ \\ ${ }^{1}$ Department of Mathematical Sciences, Florida Institute of Technology, Melbourne, FL 32901, USA \\ ${ }^{2}$ Intel Corporation, USA
}

Received March 3, 2021; Revised June 2, 2021; Accepted June 15, 2021

Cite This Paper in the following Citation Styles

(a): [1] Jewgeni H. Dshalalow, Kizza Nandyose, Ryan T. White, ”Time Sensitive Analysis Of Antagonistic Stochastic Processes And Applications To Finance And Queueing," Mathematics and Statistics, Vol.9, No.4, pp. 481-500, 2021. DOI: 10.13189/ms.2021.090408

(b): Jewgeni H. Dshalalow, Kizza Nandyose, Ryan T. White, (2021). Time Sensitive Analysis Of Antagonistic Stochastic Processes And Applications To Finance And Queueing. Mathematics and Statistics, 9(4), 481-500. DOI: 10.13189/ms.2021.090408

Copyright $@ 2021$ by authors, all rights reserved. Authors agree that this article remains permanently open access under the terms of the Creative Commons Attribution License 4.0 International License

\begin{abstract}
This paper deals with a class of antagonistic stochastic games of three players A, B, and C, of whom the first two are active players and the third is a passive player. The active players exchange hostile attacks at random times $\mathcal{T}$ $=\left\{t_{1}, t_{2}, \ldots\right\}$ of random magnitudes with each other and also with player C. Player $\mathrm{C}$ does not respond to any attacks (that are regarded as a collateral damage). There are two sustainability thresholds $M$ and $T$ are set so that when the total damages to players A and B cross $M$ and $T$, respectively, the underlying player is ruined. At some point $t_{\nu}$ (ruin time), one of the two active players will be ruined. Player C's damages are sustainable and some rebuilt. Of interest are the ruin time $t_{\nu}$ and the status of all three players upon $t_{\nu}$ as well as at any time $t$ prior to $t_{\nu}$. We obtain an analytic formula for the joint distribution of the named processes and demonstrate its closed form in various analytic and computational examples. In some situations pertaining to stock option trading, stock prices (player C) can fluctuate. So in this case, it is of interest to predict the first time when an underlying stock price drops or significantly drops so that the trader can exercise the call option prior to the drop and before maturity $T$. Player A monitors the prices upon times $\mathcal{T}$ assigning 0 damage to itself if the stock price appreciates or does not change and assumes a positive integer if the price drops. The times $\mathcal{T}$ are themselves damages to player B with threshold $T$. The "ruin" time is when threshold $M$ is crossed (i.e., there is a big price drop or a series of drops) or when the maturity $T$ expires whichever comes first. Thus a p rior a ction i $\mathrm{n}$ eeded a nd i ts $\mathrm{t}$ ime is predicted. We illustrate the applicability of the game on a number of other practical models, including queueing systems with vacations and $(\mathrm{N}, \mathrm{T})$-policy.
\end{abstract}

Keywords Random Walk, Independent and Stationary Increments Processes, Fluctuations of Stochastic Processes, Marked Point Processes, First Passage Time, Signed Marked Random Measures, Time Sensitive Analysis

\section{Introduction}

In this article we study the behavior of bivariate signed marked random measures around specified thresholds in which underlying marks are competing against each other. There are various applications of such settings found in stochastic modeling, including finance and queueing. It is very convenient to formalize the problem using some terminology of antagonistic stochastic games of several players, even though our interpretation does not include traditional game-theoretical components such as payoff functions and iterative optimization.

To serve this objective, we consider a generic game of three players, A, B, and C of whom A and B are "active players" who are involved in a periodic exchange of random hostile actions aimed at inflicting damages upon each other, while player $\mathrm{C}$ is passive being a collateral damage of the active part of the game. Player $\mathrm{C}$ periodically recovers from the blows and he does not respond to the actions from the other players. In a nutshell, such a game can be described as follows. At random times $t_{0}, t_{1}, t_{2} \ldots$, players $\mathrm{A}$ and $\mathrm{B}$ exchange with simultaneous hostile actions inflicting casualties of random magnitudes, $x_{0}, x_{1}, x_{2}, \ldots$, and $\Delta_{0}, \Delta_{1}, \Delta_{2}, \ldots$, respectively, until one of the two players becomes ruined. The latter is stipu- 
lated by two respective sustainability thresholds, $M$ and $T$, so that once (that is, upon one of the epochs $t_{j}$ 's) those thresholds are crossed due to excessive damages to at least one of the players, the game is over. Whereas players $\mathrm{A}$ and $\mathrm{B}$ are fighting against each other, player $\mathrm{C}$ collects hits of random magnitudes and although player $\mathrm{C}$ does not respond to the attacks at $t_{0}, t_{1}, t_{2}, \ldots$, he recovers from them on occasion. It is manifested by a series $\pi_{0}, \pi_{1}, \pi_{2}, \ldots$ of real-valued increments, some of which are damages (with $\pi$ 's positive) and some recoveries (with other $\pi$ 's being negative). The status of player $\mathrm{C}$ upon the game end is often of main interest.

From various useful applications of the above setting, we name three models that are motivational for studying such "games."

Model 1. Stock Option Trading. In the situation related to stock option trading, suppose a trader buys a call option for a volatile stock with maturity at time $T$. The target strategy is to exercise the option when the stock rises above the strike price at the highest stock price prior to maturity, that is, either to buy the underlying stock at the highest price and immediately sell it for profit, or sell the underlying option. Suppose the trader observes the stock randomly at times $t_{0}, t_{1}, t_{2}, \ldots$, priced at $P_{0}, P_{1}, P_{2}, \ldots$, respectively. We introduce an auxiliary process of marks $x_{0}, x_{1}, x_{2}, \ldots$ such that $x_{k}=0$ when $\pi_{k}=P_{k}-P_{k-1} \geq 0$ and $x_{k}=1$ when $\pi_{k}<0$. Because the trader is supposed to acquire the option for an appreciating stock, he/she hopes that $x_{k}$ 's are zero for a while until $x_{\nu}$ becomes strictly negative at some $t_{\nu}$. The trader opts to exercise the option at $t_{\nu}$ believing that the trend keeps changing thereafter for worse. Even a better strategy is to exercise the option at $t_{\nu-1}$ prior to the first drop at time $t_{\nu}$. Notice that $t_{\nu}$ is defined as the first moment when the stock drops for the first time or when the option expires earlier. Hence, the epoch $t_{\nu-1}$ will guarantee that $t_{\nu-1}<T$ and at the same time, the stock price will still be reasonably high. The latter may not be true though, but this needs to be investigated and evaluated predicting the status of such game upon $t_{\nu-1}$ and $t_{\nu}$. Perhaps even more reasonable would be to predict the time of a stock's sharp drop rather than just turning negative.

A somewhat similar setting has been proposed in Dshalalow $[12,13]$ and Dshalalow and Liew [16]. However, in all of these applications to stock option trading, the authors dealt with the so-called time non sensitive analysis. That is when associated functionals of the named variables did not relate to time. In contrast with the past work, the time sensitive analysis includes the information on pertinent stochastic processes providing us with their status at each moment of time $t$. In addition, the time variable screens the stock prices from the inception of the process until times $t_{\nu-1}$ and between $t_{\nu-1}$ and $t_{\nu}$. This is significantly more advanced than the results of $[12,13,16]$, but it requires a different approach and a more substantive analysis based on a double stochastic expansion of underlying functionals as it will be shown through the course of the paper.

Another article that relates to the present paper and offers applications to finance is the recent work [20] by the authors of this paper. This paper dealt with a stock price, without its competition with the time variable running up against an expiration date pertaining to option trading, with no game that requires the presence of two (active) players, and consequently, in one dimension, and thus less challenging. Yet the approach is based on time sensitive analysis that is foundational for the current work.

It has to be mentioned that most work on fluctuations of stochastic processes (including [17] by the same authors) required that they are monotone, if there a closed form of underlying functionals is targeted. With a non monotone processes such as stock prices, it turned out that treating the stock prices as the status of a passive player required the introduction of an auxiliary (active) player associated with the passive player and this is how game-theoretical setting comes out. This approach has been proposed in Dshalalow [12] and [13], although in the context of non sensitive analysis.

Model 2. Modified T-Policy in GI/G/1 Queueing System with Multiple Vacations. Suppose a single server, when he is done with a queue, goes on multiple vacations. During his absence, newly arriving customers are placed in a buffer waiting for the server to resume his service. Suppose customers' arrivals form a (delayed) renewal process $t_{0}, t_{1}, t_{2}, \ldots$, whereas the server's vacations form an independent Poisson point process upon the times $\tau_{1}, \tau_{2}, \ldots$ The server's vacations (i.e., vacation segments) are monitored upon times $t_{0}, t_{1}, t_{2}, \ldots$, so that the number of server's vacations at time $t_{k}$ is $A_{k}=$ $x_{0}+\ldots+x_{k}$, with $x_{i}$ being the number of server's vacations in interval $\left[t_{i-1}, t_{i}\right)\left(t_{-1}=0\right)$. (The latter information can be easily obtained.) The server's absence is limited by two parameters: the number of vacation trips, say $M$, and the total duration of server's absence from the system, limited by some $T>0$. Thus, the server must return to the system upon one of the epochs $t_{0}, t_{1}, t_{2}, \ldots$, say $t_{\nu}$, whichever of the two events takes place earlier: $A_{\nu} \geq M$ or $t_{\nu} \geq T$. This is a modified T-Policy in a game-theoretical setting. A basic T-Policy was introduced in the late seventies by Heyman [23] and then periodically shown up in the literature [35, 37, 40]. The present setting applies to GI/G/1/ $\infty$-type queues with multiple vacations.

Model 3. NT-Policy in M/G/1 System with Multiple Vacations. Here is another variant of the modified T-Policy. Suppose customers arrive in the system according to a marked Poisson process in an M/G/1/ $\infty$-type queue. After the queue of available customers drops below some $r$, the server leaves the system on some maintenance. Suppose the status of the system during server's maintenance period is monitored upon a delayed renewal process $t_{0}, t_{1}, t_{2}, \ldots$. The entire absence of the server is limited by two parameters $N$ and $T$, that is, if upon one of the epochs, say $t_{\nu}$, the total number of customers (including those left behind on server's departure) has accumulated to $N$ or more $(N \geq r)$ or if at $t_{\nu}$, server's vacation time exceeded $T$, the server must return to the system. There, in the system, the server will possibly rest waiting, if the queue length is still below $N$. The two policies combined are known as an NT-Policy model. There are quite a few papers on NT-Policy (cf., [2, 3, 25, 27, 39]) including one [29] by Lee et al. about a "supreme triadic" (N,D,T)-Policy. While it is of interest to find a probabilistic information about the first passage time $t_{\nu}$ and status of the system at $t_{\nu}$, it is of further importance to obtain the distribution of the queueing process at any time $t$ during 
the interval $\left[0, t_{\nu}\right)$. The latter is needed for the formation of the semi-regenerative kernel $[1,5,6]$ in order to obtain the probability distribution of the time dependent parameter queueing process $Q(t)$. This method is generally superior to a more common supplementary variables technique that, along with other limitations, requires the existence of densities for generally distributed random variables. The same need in the continuous time parameter process would be warranted for the other two models, although it is obvious that a more refined information about the underlying processes (namely, for each for $\left.t \in\left[0, t_{\nu}\right)\right)$ does not require any further apologetics and it is a stand-alone research.

The Layout of the Paper. In section 2, we proceed with a rigorous formalism of the game in the context of the functional (joint transform)

$$
\begin{aligned}
& \Phi_{\nu}(t)=E z^{A(t)} e^{-i \eta P(t)} v^{A_{\nu-1}} e^{-i \varphi P_{\nu-1}} \\
& \times e^{-\psi t_{\nu-1}} u^{A_{\nu}} e^{-i \phi P_{\nu}} e^{-\vartheta t_{\nu}} \mathbf{1}_{\left[0, t_{\nu}\right)}(t) .
\end{aligned}
$$

This functional provides us with the probabilistic status of the game for players $\mathrm{A}, \mathrm{T}$, and $\mathrm{P}$ at any time $t$ from the inception of the game until its end (exit) as well as the status of the players upon the exit $t_{\nu}$ and one step prior to the exit $t_{\nu-1}$ from the game. Section 3 undergoes a detailed analysis of this functional ending with a closed-form expression, whereas section 4 discusses special cases to support our claim of analytic tractability. We turn our attention to the stock option trading (Model 1) and, among other valuable data, predict the time of the first drop of a stock, then validate the results obtained by simulation. Due to a limited space and time, we chose not to investigate the other two models, but we plan to explore them in our forthcoming research.

Relation to the Existing Literature. Our work topically falls into fluctuations of stochastic processes, which is a widely referred to area of random walk, renewal processes, random measures, recurrent processes, Markov processes, and semiMarkov processes, to name a few [7-9, 26, 33, 36] and their applications to astronomy and biology [24], queueing [4,10,11], stochastic games [14], finance and economics [12, 13, 16, 20, 27, 30, 31, 38], physics [24, 32], and earthquakes [33]. The main focus of our work is on time sensitive fluctuations, meaning that at least some of the component processes are with continuous time parameter. Some prior work on time sensitive fluctuations is due to Dshalalow [14] (pertaining to antagonistic games), Dshalalow and Nandyose [17, 20] about one-dimensional processes, Dshalalow and White [21] on twodimensional processes. Dshalalow and White [21] studied random measures of two active nonnegative components competing against each other. However, the game was observed over arbitrary times (not adapted to the process) and consequently, the information on the status of the game was delayed. Nor did the authors of [21] consider a third, passive, non monotone component. The delayed factor yielded less tame functionals than in the present work. Finally, our processes relate to a class of signed random measures introduced in section 2 that is rarely seen in the literature (cf. [20,34]).

\section{Problem Setting}

Now all different settings brought by Models 1-3 (and even other situations) can be formalized by a game of three players, as mentioned in the introduction. A generic game under consideration is driven by the following bivariate signed random measure on a filtered probability space $\left(\Omega, \mathcal{F},\left(\mathcal{F}_{t}\right), P\right)$

$$
\begin{aligned}
\mathcal{A} \otimes \mathcal{P} \otimes \mathcal{T}= & \sum_{n=0}^{\infty}\left(x_{n}, \pi_{n}\right) \varepsilon_{t_{n}} \\
& \left(\varepsilon_{a} \text { is the Dirac point mass at point } a\right),
\end{aligned}
$$

where the marks $x_{n}$ 's are nonnegative integer- and $\pi_{n}$ 's are real-valued r.v.'s (random variables). The associated support counting measure $\sum_{n=0}^{\infty} \varepsilon_{t_{n}}$ forms a delayed renewal process. We further assume that the random measure $\mathcal{A} \otimes \mathcal{P} \otimes \mathcal{T}$ is position dependent, that is $x_{n}, \pi_{n}, \Delta_{n}=t_{n}-t_{n-1}$ are dependent. However, $\left(x_{n}, \pi_{n}, \Delta_{n}=t_{n}-t_{n-1}\right)$ is a trivariate sequence of independent random vectors and for $n=1,2, \ldots$, they are identically distributed. That being said, we assume that their joint distributions are known and given by the transforms

$\gamma_{0}(u, \phi, \vartheta)=E u^{x_{0}} e^{-i \phi \pi_{0}} e^{-\vartheta \Delta_{0}},\|u\| \leq 1, \phi \in \mathbb{R}, \operatorname{Re} \vartheta>0$.

$\gamma(u, \phi, \vartheta)=E u^{x_{1}} e^{-i \phi \pi_{1}} e^{-\vartheta \Delta_{1}},\|u\| \leq 1, \phi \in \mathbb{R}, \operatorname{Re} \vartheta>0$

Next,

$$
A_{n}:=\sum_{k=0}^{n} x_{k}, P_{n}=\sum_{k=0}^{n} \pi_{k}, t_{n}=\sum_{k=0}^{n} \Delta_{k} .
$$

Let

$$
\begin{aligned}
& \nu_{1}:=\min \left\{n=0,1, \ldots: A_{n} \geq M\right\} \\
& \nu_{2}:=\min \left\{n=0,1, \ldots: t_{n} \geq T\right\}
\end{aligned}
$$

and

$$
\nu:=\nu_{1} \wedge \nu_{2}
$$

be called the exit index of the game. The r.v. $t_{\nu}$ is referred to as the first passage time of the game.

The game is played between two active hostile players A and $\mathrm{B}$ (where $\mathrm{B}$ is meant to represent the time-related point process $\left.\left\{t_{0}, t_{1}, \ldots\right\}\right)$. The third player, $\mathrm{C}$ is passive and only takes hits, but does not respond. The game runs until one of the two active players or both are defeated on one of the epochs $t_{k}$ 's that takes place at $t_{\nu}$.

Now

$$
(A(t), P(t)):=\mathcal{A} \otimes \mathcal{P} \otimes \mathcal{T}[0, t]
$$

is the associated continuous time parameter process. The following functional of the game is of interest.

$$
\begin{array}{r}
\Phi_{\nu}(t)=E z^{A(t)} e^{-i \eta P(t)} v^{A_{\nu-1}} e^{-i \varphi P_{\nu-1}} e^{-\psi t_{\nu-1}} u^{A_{\nu}} \\
\times e^{-i \phi P_{\nu}} e^{-\vartheta t_{\nu}} \mathbf{1}_{\left[0, t_{\nu}\right)}(t),
\end{array}
$$

that is, $\Phi_{\nu}$ gives the status of the game at any time $t$ prior to $t_{\nu}$, along with the output variables $A_{\nu}, P_{\nu}$, and those one step prior to the exit at time $t_{\nu-1}$, namely, $A_{\nu-1}$ and $P_{\nu-1}$. 
For the sequel, we set

$$
A_{-1}=t_{-1}=P_{-1}=0 .
$$

We further notice that $(A(t), P(t))$ is piece-wise linear jump process and it is constant on each time subinterval $\left[t_{k-1}, t_{k}\right)$.

\section{Analysis of $\Phi_{\nu}$}

To deal with $\Phi_{\nu}$, we break it into three functionals $\Phi_{\nu_{1}<\nu_{2}}, \Phi_{\nu_{1}>\nu_{2}}$, and $\Phi_{\nu_{1}=\nu_{2}}$ treated in this section separately.

\subsection{Player B defeats player A}

In this subsection, we consider the game adapted to the confined filtration $\mathcal{F}_{t} \cap\left\{\nu_{1}<\nu_{2}\right\}$. The associated functional reads

$$
\begin{gathered}
\Phi_{\nu_{1}<\nu_{2}}(t)=E z^{A(t)} e^{-i \eta P(t)} v^{A_{\nu_{1}-1}} e^{-i \varphi P_{\nu_{1}-1}} e^{-\psi t_{\nu_{1}-1}} \\
\times u^{A_{\nu_{1}}} e^{-i \phi P_{\nu_{1}}} e^{-\vartheta t_{\nu_{1}}} \mathbf{1}_{\left[0, t_{\nu_{1}}\right)}(t) \mathbf{1}_{\left\{\nu_{1}<\nu_{2}\right\}} \\
=\sum_{k=0}^{\infty} \sum_{n>k} E\left[z^{A(t)} e^{-i \eta P(t)} v^{A_{k-1}} e^{-i \varphi P_{k-1}}\right. \\
\times e^{-\psi t_{k-1}} u^{A_{k}} e^{-i \phi P_{k}} e^{-\vartheta t_{k}} \mathbf{1}_{\left[0, t_{k}\right)}(t) \\
\left.\times \mathbf{1}_{\{k\}}\left(\nu_{1}\right) \mathbf{1}_{\{n\}}\left(\nu_{2}\right)\right] .
\end{gathered}
$$

We partition the interval $\left[0, t_{k}\right)$ into $\left[0, t_{k}\right)=$ $\bigcup_{j=0}^{k}\left[t_{k-j-1}, t_{k-j}\right)$ under (2.7) to yield

$$
\begin{aligned}
\Phi_{\nu_{1}<\nu_{2}}(t)= & \sum_{k=0}^{\infty} \sum_{n>k} \sum_{j=0}^{k} E\left[z^{A(t)} e^{-i \eta P(t)} v^{A_{k-1}} e^{-i \varphi P_{k-1}}\right. \\
& \times e^{-\psi t_{k-1}} u^{A_{k}} e^{-i \phi P_{k}} e^{-\vartheta t_{k}} \\
\times & \left.\mathbf{1}_{\left[t_{k-j-1}, t_{k-j}\right)}(t) \mathbf{1}_{\{k\}}\left(\nu_{1}\right) \mathbf{1}_{\{n\}}\left(\nu_{2}\right)\right] .
\end{aligned}
$$

In our next efforts to unfold $\Phi_{\nu_{1}<\nu_{2}}$ we will apply methods of discrete and continuous operational calculus. We will use an approach previously developed by Dshalalow [14] and further explored in Dshalalow and White [21] and referred to as a stochastic expansion. To this end, we define two sequences of exit indices

$$
\nu_{1}(p)=\inf \left\{n=0,1, \ldots: A_{n}>p\right\}, p=0,1, \ldots,
$$

and

$$
\nu_{2}(q)=\inf \left\{n=0,1, \ldots: t_{n} \geq q\right\}, q \geq 0 .
$$

Then, for any pair $(p, q) \in \mathbb{N}_{0} \times \mathbb{R}_{+}$, let

$$
\nu(p, q)=\nu_{1}(p) \wedge \nu_{2}(q) .
$$

Note that $\nu_{1}=\nu_{1}(M-1)$ and $\nu_{2}=\nu_{2}(T)$. Now consider the associated extended family of functionals $\left\{\Phi_{\nu_{1}(p)<\nu_{2}(q)}(t):(p, q) \in \mathbb{N}_{0} \times \mathbb{R}_{+}\right\}$in the form of

$$
\begin{aligned}
\Phi_{\nu_{1}(p)<\nu_{2}(q)} & (t)=E\left[z^{A(t)} e^{-i \eta P(t)} v^{A_{\nu_{1}(p)-1}} e^{-i \varphi P_{\nu_{1}(p)-1}}\right. \\
\times & e^{-\psi t_{\nu_{1}(p)-1}} u^{A_{\nu_{1}(p)}} e^{-i \phi P_{\nu_{1}(p)}} \\
\times & \left.e^{-\vartheta t_{\nu_{1}(p)}} \mathbf{1}_{\left[0, t_{\nu_{1}(p)}\right)}(t) \mathbf{1}_{\left\{\nu_{1}(p)<\nu_{2}(q)\right\}}\right]
\end{aligned}
$$

to which we apply the transforms $D_{p}$ and $\mathcal{L C}_{q}$ defined as follows.

$$
\begin{aligned}
f^{*}(x, q)=D_{p}\{f(p, q)\}(x, q) \\
:=\sum_{p=0}^{\infty} x^{p} f(p, q)(1-x),\|x\|<1,
\end{aligned}
$$

where $f$ is a complex-valued analytic function with the domain $\mathbb{N}_{0} \times \mathbb{R}_{+}$. The inverse of $D_{p}$ is the so-called $\mathcal{D}$-operator previously introduced in Dshalalow (cf., [15]):

$$
\begin{aligned}
f(k, q) & =\mathcal{D}_{x}^{k} f^{*}(x, q) \\
& =\left\{\begin{array}{cc}
\lim _{x \rightarrow 0} \frac{1}{k !} \frac{\partial^{k}}{\partial x^{k}}\left[\frac{1}{1-x} f^{*}(x, q)\right], & k \geq 0 \\
0, & k<0 .
\end{array}\right.
\end{aligned}
$$

The $\mathcal{L C}$ denotes the Laplace-Carson transform defined as

$$
\mathcal{L C}_{q} \varphi(y)=y \int_{q=0}^{\infty} e^{-y q} \varphi(q) d q, \operatorname{Re} y>0 .
$$

The Laplace-Carson transform follows the familiar pattern of the Laplace transform whose inverse is

$$
\mathcal{L C}_{y}^{-1}(\cdot)=\mathcal{L}_{y}^{-1}\left[\frac{1}{y} \cdot\right]
$$

with $\mathcal{L}$ standing for the Laplace transform. Together, they form the composition

$$
\mathcal{L C}_{q} D_{p}:=\mathcal{L C}_{q} \circ D_{p}
$$

Thus, if

$$
\hat{f}(x, y)=\mathcal{L C}_{q} D_{p} f(p, q)(x, y)
$$

then

$$
f(k, q)=\mathcal{D}_{x}^{k} \mathcal{L C}_{y}^{-1}(\hat{f}(x, y))(k, q) .
$$

Note that the transforms can be applied in any order.

Now with (3.7-3.13) in mind, the functional $\Phi_{\nu_{1}<\nu_{2}}(t)$, after the $(p, q)$-expansion and the use of operator $\mathcal{L C}_{q} D_{p}$ turns to

$$
\begin{aligned}
& \hat{\Phi}_{\nu_{1}<\nu_{2}}(t, x, y) \\
& =\mathcal{L C}_{q} D_{p} \sum_{k=0}^{\infty} \sum_{j=0}^{k} \sum_{n>k} E\left[z^{A(t)} e^{-i \eta P(t)} v^{A_{k-1}} e^{-i \varphi P_{k-1}}\right. \\
& e^{-\psi t_{k-1}} u^{A_{k}} e^{-i \phi P_{k}} e^{-\vartheta t_{k}} \mathbf{1}_{\left[t_{k-j-1}, t_{k-j}\right)}(t) \mathbf{1}_{\{k\}}\left(\nu_{1}\right) \\
& \left.\quad \times \mathbf{1}_{\{n\}}\left(\nu_{2}\right)\right](x, y) \\
& =\sum_{k=0}^{\infty} \sum_{j=0}^{k} \sum_{n>k} E\left[z^{A(t)} e^{-i \eta P(t)} v^{A_{k-1}} e^{-i \varphi P_{k-1}} e^{-\psi t_{k-1}}\right. \\
& \quad \times u^{A_{k}} e^{-i \phi P_{k}} e^{-\vartheta t_{k}} \mathbf{1}_{\left[t_{k-j-1}, t_{k-j}\right)}(t) \\
& \left.\quad \times \mathcal{L C}_{q} D_{p}\left[\mathbf{1}_{\{k\}}\left(\nu_{1}\right) \mathbf{1}_{\{n\}}\left(\nu_{2}\right)\right](x, y)\right]
\end{aligned}
$$

By Fubini's theorem and from 


$$
\begin{aligned}
\mathcal{L C}_{q} D_{p}\left[\mathbf{1}_{\{k\}}\left(\nu_{1}\right) \mathbf{1}_{\{n\}}\left(\nu_{2}\right)\right](x, y) \\
=\left(x^{A_{k-1}}-x^{A_{k}}\right)\left(e^{-y t_{n-1}}-e^{-y t_{n}}\right),
\end{aligned}
$$

(cf., Dshalalow [15]), we have from (3.14),

$$
\Phi_{\nu_{1}<\nu_{2}}(t, x, y)=\sum_{k=0}^{\infty} \sum_{j=0}^{k} \Phi_{j k}(t, x, y)
$$

where

$$
\begin{array}{r}
\Phi_{j k}(t, x, y)=\sum_{n>k} E\left[z^{A(t)} e^{-i \eta P(t)} v^{A_{k-1}} e^{-i \varphi P_{k-1}}\right. \\
\times e^{-\psi t_{k-1}} u^{A_{k}} e^{-i \phi P_{k}} e^{-\vartheta t_{k}} \mathbf{1}_{\left[t_{k-j-1}, t_{k-j}\right)}(t) \\
\left.\times\left(x^{A_{k-1}}-x^{A_{k}}\right)\left(e^{-y t_{n-1}}-e^{-y t_{n}}\right)\right] .
\end{array}
$$

To further continue with $\hat{\Phi}_{\nu_{1}<\nu_{2}}(t, x, y)$ we break (3.163.17) into four separate cases. We start with

Case $(i) .0<j<k$.

$$
\begin{gathered}
\Phi_{j k}(t, x, y)=\sum_{n>k} E\left[z^{A_{k-j-1}} e^{-i \eta P_{k-j-1}} v^{A_{k-1}} e^{-i \varphi P_{k-1}}\right. \\
\times e^{-\psi t_{k-1}} u^{A_{k}} e^{-i \phi P_{k}} e^{-\vartheta t_{k}} \mathbf{1}_{\left[t_{k-j-1}, t_{k-j}\right)}(t) \\
\left.\times\left(x^{A_{k-1}}-x^{A_{k}}\right)\left(e^{-y t_{n-1}}-e^{-y t_{n}}\right)\right] \\
=E\left[\mathbf{1}_{\left[t_{k-j-1}, t_{k-j}\right)}(t)(u v z x)^{A_{k-j-1}} e^{-i(\varphi+\phi+\eta) P_{k-j-1}}\right. \\
\times e^{-\left(\vartheta+\pi_{k-j}+\ldots+\pi_{k-1}\right)} \\
\left.\times e^{-(\vartheta+\psi+y)\left(\Delta_{k-j}+\ldots+\Delta_{k-1}\right)}\right] \\
\times E\left[u^{x_{k}} e^{-i \phi \pi_{k}} e^{-(\vartheta+y) \Delta_{k}}\left(1-x^{x_{k}}\right)\right] \\
\times \sum_{n>k} E\left[e^{-y\left(\Delta_{k+1}+\ldots+\Delta_{n-1}\right)}\left(1-e^{-y \Delta_{n}}\right)\right] .
\end{gathered}
$$

From (2.3),

$$
\begin{aligned}
\sum_{n>k} E & {\left[e^{-y\left(\Delta_{k+1}+\ldots+\Delta_{n-1}\right)}\left(1-e^{-y \Delta_{n}}\right)\right] } \\
& =\sum_{n>k} \gamma^{n-k-1}(1,0, y)[1-\gamma(1,0, y)]=1
\end{aligned}
$$

The latter holds true, because $\|\gamma(1,0, y)\|<1$ due to the restriction $\operatorname{Re} y>0$ in (3.9) (imposed on $y$ for different reasons). Thus, from (3.19),

$$
\begin{gathered}
\Phi_{j k}(t, x, y) \\
=E\left[\mathbf{1}_{\left[t_{k-j-1}, t_{k-j}\right)}(t)(u v z x)^{A_{k-j-1}} e^{-i(\varphi+\phi+\eta) P_{k-j-1}}\right. \\
\left.\times e^{-(\vartheta+\psi+y) t_{k-j-1}}(u v x)^{x_{k-j}} e^{-i(\varphi+\phi) \pi_{k-j}} e^{-(\vartheta+\psi+y) \Delta_{k-j}}\right] \\
\times E\left[(u v x)^{x_{k-j+1}+\ldots+x_{k-1}} e^{-i(\varphi+\phi)\left(\pi_{k-j+1}+\ldots+\pi_{k-1}\right)}\right. \\
\left.\times e^{-(\vartheta+\psi+y)\left(\Delta_{k-j+1}+\ldots+\Delta_{k-1}\right)}\right] \\
\times E\left[u^{x_{k}} e^{-i \phi \pi_{k}} e^{-(\vartheta+y) \Delta_{k}}\left(1-x^{x_{k}}\right)\right]
\end{gathered}
$$

(such factorization is due to $\Delta_{k-j}$ 's dependence on $\left[t_{k-j-1}, t_{k-j}\right)$ and $x_{k-j}$ and $\pi_{k-j}$ dependence on $\left.\Delta_{k-j}\right)$ arriving at

$$
\Phi_{j k}(t, x, y)=\Phi_{j k}(t, x, y)=F_{j k}(t, x, y) f_{j}(x, y) .
$$

Here,

$$
\begin{gathered}
F_{j k}(t, x, y)=E\left[\mathbf{1}_{\left[t_{k-j-1}, t_{k-j-1}+\Delta_{k-j}\right)}(t)(u v z x)^{A_{k-j-1}}\right. \\
\times e^{-i(\varphi+\phi+\eta) P_{k-j-1}} e^{-(\vartheta+\psi+y) t_{k-j-1}} \\
\left.\times(u v x)^{x_{k-j}} e^{-i(\varphi+\phi) \pi_{k-j}} e^{-(\vartheta+\psi+y) \Delta_{k-j}}\right]
\end{gathered}
$$

and, with (2.3),

$$
\begin{aligned}
f_{j}(x, y)= & \gamma^{j-1}(u v x, \varphi+\phi, \vartheta+\psi+y) \\
& \times[\gamma(u, \phi, \vartheta+y)-\gamma(u x, \phi, \vartheta+y)]
\end{aligned}
$$

At this point, we turn to a further transform applied to $\hat{\Phi}_{\nu}(t, x, y)$ and subsequently to $\hat{\Phi}_{\nu_{1}<\nu_{2}}(t, x, y)$ and all pertinent terms and factors containing time $t$, namely, the Laplace transform with respect to $t$.

$$
\Phi_{\nu}^{*}(\theta, x, y):=\int_{t=0}^{\infty} e^{-\theta t} \hat{\Phi}_{\nu}(t, x, y) d t
$$

Hence, from (3.22),

$$
\begin{array}{r}
F_{j k}^{*}(\theta, x, y)=\int_{t=0}^{\infty} e^{-\theta t} F_{k j}(t, x, y) d t \\
=\sum_{a=0}^{\infty}(u v z x)^{a} \sum_{\xi=0}^{\infty}(u v x)^{\xi} \int_{p=-\infty}^{\infty} e^{-i(\varphi+\phi+\eta) p} \\
\times \int_{q=-\infty}^{\infty} e^{-i(\varphi+\phi) q} \int_{s=0}^{\infty} e^{-(\vartheta+\psi+y) s} \\
\times \int_{\delta=0}^{\infty} e^{-(\vartheta+\psi+y) \delta}\left(\int_{t=s}^{s+\delta} e^{-\theta t} d t\right) \\
\times P_{A_{k-j-1} \otimes P_{k-j-1} \otimes t_{k-j-1} \otimes x_{k-j} \otimes \pi_{k-j} \otimes \Delta_{k-j}} \\
(a, d p, d s, \xi, d s, d \delta),
\end{array}
$$


and after straightforward calculations, with (2.2-2.3),

$$
\begin{gathered}
F_{j k}^{*}(\theta, x, y)=\frac{1}{\theta} \gamma_{0}(u v z x, \varphi+\phi+\eta, \theta+\vartheta+\psi+y) \\
\times \gamma^{k-j-1}(u v z x, \varphi+\phi+\eta, \theta+\vartheta+\psi+y) \\
\times[\gamma(u v x, \varphi+\phi, \vartheta+\psi+y) \\
\quad-\gamma(u v x, \varphi+\phi, \theta+\vartheta+\psi+y)] .
\end{gathered}
$$

For now and in the sequel, it will be easier to operate under the following abbreviations:

$$
\begin{gathered}
\gamma=\gamma(u v z x, \varphi+\phi+\eta, \theta+\vartheta+\psi+y), \gamma_{0} \\
=\gamma_{0}(u v z x, \varphi+\phi+\eta, \theta+\vartheta+\psi+y),
\end{gathered}
$$

$$
\begin{aligned}
\hat{\gamma}=\gamma(u v x, \varphi+\phi, \vartheta+\psi+y), \hat{\gamma}(\theta) & \\
& =\gamma(u v x, \varphi+\phi, \theta+\vartheta+\psi+y),
\end{aligned}
$$

$$
g=\gamma(u x, \phi, \vartheta+y), g_{1}=\gamma(u, \phi, \vartheta+y)
$$

Thus, (3.26) reads

$$
F_{j k}^{*}(\theta, x, y)=\frac{1}{\theta} \gamma_{0} \gamma^{k-j-1}[\hat{\gamma}-\hat{\gamma}(\theta)]
$$

and $f_{j}(x, y)=\hat{\gamma}^{j-1}\left[g_{1}-g\right]$.

Now summing up $F_{j k}^{*}(\theta, x, y) f_{j}(x, y)$ for all $0<j<k$ we get

$$
\sum_{k=1}^{\infty} \sum_{j=1}^{k-1} \Phi_{j k}^{*}(\theta, x, y)=\gamma_{0} \frac{g_{1}-g}{(1-\gamma)(1-\hat{\gamma})} \Gamma \gamma
$$

where

$$
\Phi_{j k}^{*}(\theta, x, y):=\int_{t=0}^{\infty} e^{-\theta t} \Phi_{j k}(t, x, y) d t
$$

and

$$
\Gamma \gamma:=\frac{1}{\theta}[\hat{\gamma}-\hat{\gamma}(\theta)]
$$

Now formula (3.31) is due to the convergence of associated geometric series guaranteed by

$$
\|\gamma\|<1 \text { and }\|\hat{\gamma}\|<1
$$

(cf. Dshalalow and Nandyose $[17,20])$.
Case $(i i) \cdot j=0, k>0$.

$$
\begin{aligned}
& \Phi_{0 k}(t, x, y)=\sum_{n>k} E\left[z^{A(t)} e^{-i \eta P(t)} v^{A_{k-1}} e^{-i \varphi P_{k-1}}\right. \\
& \times e^{-\psi t_{k-1}} u^{A_{k}} e^{-i \phi P_{k}} e^{-\vartheta t_{k}} \mathbf{1}_{\left[t_{k-1}, t_{k}\right)}(t)\left(x^{A_{k-1}}-x^{A_{k}}\right) \\
& \left.\times\left(e^{-y t_{n-1}}-e^{-y t_{n}}\right)\right] \\
& =E\left[z^{A_{k-1}} e^{-i \eta P_{k-1}} v^{A_{k-1}} e^{-i \varphi P_{k-1}} e^{-\psi t_{k-1}} u^{A_{k}} e^{-i \phi P_{k}}\right. \\
& \times e^{-\vartheta t_{k}} \mathbf{1}_{\left[t_{k-1}, t_{k}\right)}(t)\left(x^{A_{k-1}}-x^{A_{k}}\right) \\
& \left.\times e^{-y t_{k-1}} e^{-y \Delta_{k}}\right] \sum_{n>k} E\left[e^{-y \Delta_{k+1}+\ldots+\Delta_{n-1}}\left(1-e^{-y \Delta_{n}}\right)\right] \\
& =E\left[(u v z x)^{A_{k-1}} e^{-i(\varphi+\phi+\eta) P_{k-1}} e^{-(\psi+\vartheta+y) t_{k-1}}\right. \\
& \left.\times u^{x_{k}}\left(1-x^{x_{k}}\right) e^{-i \phi \pi_{k}} e^{-(\vartheta+y) \Delta_{k}} \mathbf{1}_{\left[t_{k-1}, t_{k-1}+\Delta_{k}\right)}(t)\right] .
\end{aligned}
$$

Then applying the Laplace transform and unfolding the expectation, from (3.35),

$$
\begin{gathered}
\Phi_{0 k}^{*}(\theta, x, y)=\sum_{a=0}^{\infty}(u v z x)^{a} \sum_{\xi=0}^{\infty}\left[u^{\xi}-(u x)^{\xi}\right] \\
\times \int_{p=-\infty}^{\infty} e^{-i(\varphi+\phi+\eta) p} \int_{q=-\infty}^{\infty} e^{-i \phi q} \int_{s=0}^{\infty} e^{-(\vartheta+\psi+y) s} \\
\times \int_{\delta=0}^{\infty} e^{-(\vartheta+y) \delta} \int_{t=s}^{s+\delta} e^{-\theta t} d t \times \int_{q=-\infty}^{\infty} e^{-i \phi q} \\
\times \int_{s=0}^{\infty} e^{-(\vartheta+\psi+y) s} \int_{\delta=0}^{\infty} e^{-(\vartheta+y) \delta} \int_{t=s}^{s+\delta} e^{-\theta t} d t \\
P_{A_{k-1} \otimes P_{k-1} \otimes t_{k-1} \otimes x_{k} \otimes \pi_{k} \otimes \Delta_{k}}(a, d p, d s, \xi, d s, d \delta) \\
=\frac{1}{\theta} \gamma_{0} \gamma^{k-1}\left[g_{1}-g-g_{1}(\theta)+g(\theta)\right]
\end{gathered}
$$

with

$$
g(\theta)=\gamma(u x, \phi, \theta+\vartheta+y), g_{1}(\theta)=\gamma(u, \phi, \theta+\vartheta+y)
$$

Summing up $\Phi_{0 k}^{*}(\theta, x, y)$ over all $k=1,2, \ldots$, under (3.34) gives

$$
\sum_{k=1}^{\infty} \Phi_{0 k}^{*}(\theta, x, y)=\frac{\gamma_{0}}{1-\gamma} \frac{1}{\theta}\left[g_{1}-g-g_{1}(\theta)+g(\theta)\right]
$$

Case $(i i i) \cdot j=k>0$. 


$$
\begin{gathered}
\Phi_{k k}(t, x, y)=\sum_{n>k} E\left[z^{A(t)} e^{-i \eta P(t)} v^{A_{k-1}} e^{-i \varphi P_{k-1}} e^{-\psi t_{k-1}}\right. \\
\times u^{A_{k}} e^{-i \phi P_{k}} e^{-\vartheta t_{k}} \mathbf{1}_{\left[t_{-1}, t_{0}\right)}(t)\left(x^{A_{k-1}}-x^{A_{k}}\right) \\
\left.\left(e^{-y t_{n-1}}-e^{-y t_{n}}\right)\right] \\
=E\left[\mathbf{1}_{\left[t_{-1}, t_{0}\right)}(t)(u v z x)^{A_{-1}} e^{-i(\varphi+\phi+\eta) P_{-1}} e^{-(\vartheta+\psi+y) t_{-1}}\right. \\
\times(u v x)^{x_{0}+\ldots+x_{k-1}} \\
\left.\times e^{-i(\varphi+\phi)\left(\pi_{0}+\ldots+\pi_{k-1}\right)} e^{-(\vartheta+\psi+y)\left(\Delta_{0}+\ldots+\Delta_{k-1}\right)}\right] \\
\times E\left[u^{x_{k}} e^{-i \phi \pi_{k}} e^{-(\vartheta+y) \Delta_{k}}\left(1-x^{x_{k}}\right)\right] \sum_{n>k} \\
\times E\left[e^{-y\left(\Delta_{k+1}+\ldots+\Delta_{n-1}\right)}\left(1-e^{-y \Delta_{n}}\right)\right]
\end{gathered}
$$

Next with $t_{-1}=A_{-1}=P_{-1}=0$ and from (3.19),

implying that

$$
\begin{gathered}
\Phi_{k k}(t, x, y)=E\left[\mathbf{1}_{\left[0, t_{0}\right)}(t)(u v x)^{x_{0}+\ldots+x_{k-1}}\right. \\
\left.\times e^{-i(\varphi+\phi)\left(\pi_{0}+\ldots+\pi_{k-1}\right)} e^{-(\vartheta+\psi+y)\left(\Delta_{0}+\ldots+\Delta_{k-1}\right)}\right] \\
\times E\left[u^{x_{k}} e^{-i \phi \pi_{k}} e^{-(\vartheta+y) \Delta_{k}}\left(1-x^{x_{k}}\right)\right] \\
=E\left[\mathbf{1}_{\left[0, t_{0}\right)}(t)(u v x)^{A_{0}} e^{-i(\varphi+\phi) P_{0}} e^{-(\vartheta+\psi+y) t_{0}}\right] \\
\times E\left[(u v x)^{x_{1}+\ldots+x_{k-1}} e^{-i(\varphi+\phi)\left(\pi_{1}+\ldots+\pi_{k-1}\right)}\right. \\
\left.\times e^{-(\vartheta+\psi+y)\left(\Delta_{1}+\ldots+\Delta_{k-1}\right)} e^{-i \phi \pi_{k}} e^{-(\vartheta+y) \Delta_{k}}\left(u^{x_{k}}-(u x)^{x_{k}}\right)\right] .
\end{gathered}
$$

$$
\begin{aligned}
& \text { So } \\
& \begin{aligned}
& \Phi_{k k}^{*}(t, x, y)=\sum_{a}(u v x)^{a} \int_{p=0}^{\infty} e^{-i(\varphi+\phi) p} \int_{s=0}^{\infty} e^{-(\vartheta+\psi+y) s} \\
& \times \int_{0}^{s} e^{-\theta t} d t P_{A_{0} \otimes P_{0} \otimes t_{0}}(a, d p, d s) \\
& E[(u v x)^{x_{1}+\ldots+x_{k-1}} e^{-i(\varphi+\phi)\left(\pi_{1}+\ldots+\pi_{k-1}\right)} \\
&\left.\times e^{-(\vartheta+\psi+y)\left(\Delta_{1}+\ldots+\Delta_{k-1}\right)} e^{-i \phi \pi_{k}} e^{-(\vartheta+y) \Delta_{k}}\left(u^{x_{k}}-(u x)^{x_{k}}\right)\right] \\
&=\frac{1}{\theta}\left[\gamma_{0}(u v x, \varphi+\phi, \theta+\vartheta+\psi+y)\right. \\
&\left.\quad-\gamma_{0}(u v x, \varphi+\phi, \vartheta+\psi+y)\right] \\
& \times \gamma^{k-1}(u v x, \varphi+\phi, \vartheta+\psi+y) \\
& \times[\gamma(u, \phi, \vartheta+y)-\gamma(u x, \phi, \vartheta+y)] \\
& \quad=\frac{1}{\theta}\left[\hat{\gamma}_{0}-\hat{\gamma}_{0}(\theta)\right] \hat{\gamma}^{k-1}\left[g_{1}-g\right] . \quad(3.40)
\end{aligned}
\end{aligned}
$$

By (3.34) and summing up $\Phi_{k k n}^{*}(\theta, x, y)$ over all pertinent $k$ 's gives

$$
\sum_{k=1}^{\infty} \Phi_{k k}^{*}(\theta, x, y)=\frac{g_{1}-g}{1-\hat{\gamma}}\left[\hat{\gamma}_{0}-\hat{\gamma}_{0}(\theta)\right]=\frac{g_{1}-g}{1-\hat{\gamma}} \Gamma \gamma_{0}
$$

and so we are done with case $($ iii $)$.

Case $(\boldsymbol{i v}) \cdot j=k=0$.

$$
\begin{gathered}
\Phi_{00}(t, x, y)=\sum_{n>0} E\left[z^{A(t)} e^{-i \eta P(t)} v^{A_{-1}} e^{-i \varphi P_{-1}}\right. \\
\times e^{-\psi t_{-1}} u^{A_{0}} e^{-i \phi P_{0}} e^{-\vartheta t_{0}} \mathbf{1}_{\left[t_{-1}, t_{0}\right)}(t) \\
\left.\times\left(x^{A_{-1}}-x^{A_{0}}\right)\left(e^{-y t_{n-1}}-e^{-y t_{n}}\right)\right] \\
=E\left[z^{A-1} e^{-i \eta P_{-1}} v^{A_{-1}} e^{-i \varphi P_{-1}} e^{-\psi t_{-1}} u^{A_{0}} e^{-i \phi P_{0}}\right. \\
\quad \times e^{-\vartheta t_{0}} \mathbf{1}_{\left[t_{-1}, t_{0}\right)}(t)\left(x^{A_{-1}}-x^{A_{0}}\right) \\
\left.\times e^{-y t_{-1}} e^{-y \Delta_{0}}\right] \sum_{n>0} E\left[e^{-y \Delta_{1}+\ldots+\Delta_{n-1}}\left(1-e^{-y \Delta_{n}}\right)\right],
\end{gathered}
$$

where $\Delta_{0}=t_{0}$. Thus,

$\Phi_{00}(t, x, y)=E\left[u^{A_{0}} e^{-i \phi P_{0}} e^{-(\vartheta+y) t_{0}} \mathbf{1}_{\left[0, t_{0}\right)}(t)\left(1-x^{A_{0}}\right)\right]$

and

$$
\begin{gathered}
\Phi_{00}^{*}(t, x, y) \\
=\sum_{a}\left(u^{a}-(u x)^{a}\right) \int_{p=0}^{\infty} e^{-i \phi p} \int_{s=0}^{\infty} e^{-(\vartheta+y) s} \\
\times \int_{0}^{s} e^{-\theta t} d t P_{A_{0} \otimes P_{0} \otimes t_{0}}(a, d p, d s) \\
=\frac{1}{\theta}\left[g_{01}-g_{0}-g_{01}(\theta)+g_{0}(\theta)\right] .
\end{gathered}
$$


Finally, summing up the respective components in all four cases yields

$$
\begin{aligned}
& \Phi_{\nu_{1}<\nu_{2}}^{*}(\theta, x, y)=\sum_{k=1}^{\infty} \sum_{j=1}^{k-1} \Phi_{j k}^{*}(\theta, x, y) \\
& \quad+\sum_{k=1}^{\infty} \Phi_{0 k}^{*}(\theta, x, y)+\sum_{k=1}^{\infty} \Phi_{k k}^{*}(\theta, x, y)+\Phi_{00}^{*}(t, x, y) \\
& =\gamma_{0} \frac{g_{1}-g}{(1-\gamma)(1-\hat{\gamma})} \Gamma \gamma+\frac{\gamma_{0}}{1-\gamma} \frac{1}{\theta}\left[g_{1}-g-g_{1}(\theta)+g(\theta)\right] \\
& \quad+\frac{g_{1}-g}{1-\hat{\gamma}} \Gamma \gamma_{0}+\frac{1}{\theta}\left[g_{01}-g_{0}-g_{01}(\theta)+g_{0}(\theta)\right] .
\end{aligned}
$$

\subsection{Player A defeats player B}

In the functional

$$
\begin{gathered}
\Phi_{\nu_{2}<\nu_{1}}(t)=E\left[z^{A(t)} e^{-i \eta P(t)} v^{A_{\nu_{2}}-1} e^{-i \varphi P_{\nu_{2}-1}} e^{-\psi t_{\nu_{2}-1}}\right. \\
\left.u^{A_{\nu_{2}}} e^{-i \phi P_{\nu_{2}}} e^{-\vartheta t_{\nu_{2}}} \mathbf{1}_{\left[0, t_{\nu_{2}}\right)}(t) \mathbf{1}_{\left\{\nu_{2}<\nu_{1}\right\}}\right]
\end{gathered}
$$

and its Laplace transform we need to reverse the roles of $x$ and $y$, but only in those terms where the representation of $x$ and $y$ is asymmetric, e.g., $g(1, y), g(1, y, \theta)$ and subsequently $\Gamma g$ and $\Gamma g(\theta)$, with $g_{2}=g(x, 0)=\gamma(u x, \phi, \vartheta)$, $g_{2}(\theta)=g(x, 0, \theta)=\gamma(u x, \phi, \theta+\vartheta)$ thus, arriving at

$$
\begin{gathered}
\Phi_{\nu_{1}<\nu_{2}}^{*}(\theta, x, y)=\gamma_{0} \frac{g_{2}-g}{(1-\gamma)(1-\hat{\gamma})} \Gamma \gamma \\
+\frac{\gamma_{0}}{1-\gamma} \frac{1}{\theta}\left[g_{2}-g-g_{2}(\theta)+g(\theta)\right] \\
+\frac{g_{2}-g}{1-\hat{\gamma}} \Gamma \gamma_{0}+\frac{1}{\theta}\left[g_{02}-g_{0}-g_{02}(\theta)+g_{0}(\theta)\right]
\end{gathered}
$$

\subsection{Both players are ruined upon the first passage time}

In this third alternative we have

$$
\begin{aligned}
& \Phi_{\nu_{1}=\nu_{2}}(t)=E\left[z^{A(t)} e^{-i \eta P(t)} v^{A_{\nu_{1}-1}}\right. \\
\times & \left.e^{-i \varphi P_{\nu_{1}-1}} e^{-\psi t_{\nu_{1}-1}} u^{A_{\nu_{1}}} e^{-i \phi P_{\nu_{1}}} e^{-\vartheta t_{\nu_{1}}} \mathbf{1}_{\left[0, t_{\nu_{1}}\right)}(t) \mathbf{1}_{\left\{\nu_{1}=\nu_{2}\right\}}\right]
\end{aligned}
$$

and

$$
\hat{\Phi}_{\nu_{1}=\nu_{2}}(t, x, y)=D_{p} \mathcal{L} \mathcal{C}_{q} \Phi_{\nu_{2}=\nu_{1}}(t)=\sum_{k=0}^{\infty} \sum_{j=0}^{k} \Phi_{j k}(t, x, y),
$$

where

$$
\begin{aligned}
& \Phi_{j k}(t, x, y)=E\left[z^{A(t)} e^{-i \eta P(t)}\right. \\
& \times v^{A_{k-1}} e^{-i \varphi P_{k-1}} e^{-\psi t_{k-1}} u^{A_{k}} e^{-i \phi P_{k}} e^{-\vartheta t_{k}} \\
& \left.\times \mathbf{1}_{\left[t_{k-j-1}, t_{k-j}\right)}(t)\left(x^{A_{k-1}}-x^{A_{k}}\right)\left(e^{-y t_{k-1}}-e^{-y t_{k}}\right)\right] .
\end{aligned}
$$

Then we break (3.48) into four cases below.
Case $(i) .0<j<k$. Here,

$$
\begin{gathered}
\Phi_{j k}(t, x, y)=\sum_{k=1}^{\infty} \sum_{j=1}^{k-1} E\left[\mathbf{1}_{\left[t_{k-j-1}, t_{k-j}\right)}(t)\right. \\
\times(u v z x)^{A_{k-j-1}}(u v x)^{x_{k-j}}(u v x)^{x_{k-j+1}+\ldots+x_{k-1}} u^{x_{k}}\left(1-x^{x_{k}}\right) \\
\times e^{-i(\varphi+\phi+\eta) P_{k-j-1}} e^{-i(\varphi+\phi) \pi_{k-j}} e^{-i(\varphi+\phi)\left(\pi_{k-j+1}+\ldots+\pi_{k-1}\right)} \\
\times e^{-i \phi \pi_{k}} e^{-(\vartheta+\psi+y) t_{k-j-1}} e^{-(\vartheta+\psi+y)\left(\Delta_{k-j}+\ldots+\Delta_{k-1}\right)} \\
\left.\times e^{-\vartheta \Delta_{k}}\left(1-e^{-y \Delta_{k}}\right)\right] \\
=\sum_{k=0}^{\infty} \sum_{j=0}^{k} F_{j k}(t, x, y) f_{j}(x, y)
\end{gathered}
$$

where

$$
\begin{aligned}
& F_{j k}(t, x, y)=E\left[\mathbf{1}_{\left[t_{k-j-1}, t_{k-j}\right)}(t)\right. \\
& \times(u v z x)^{A_{k-j-1}} e^{-i(\varphi+\phi+\eta) P_{k-j-1}} e^{-(\vartheta+\psi+y) t_{k-j-1}} \\
& \left.\quad \times(u v x)^{x_{k-j}} e^{-i(\varphi+\phi) \pi_{k-j}} e^{-(\vartheta+\psi+y) \Delta_{k-j}}\right]
\end{aligned}
$$

and

$$
\begin{aligned}
& f_{j}(x, y)=E(u v x)^{x_{k-j+1}+\ldots+x_{k-1}} \\
& \times e^{-i(\varphi+\phi)\left(\pi_{k-j+1}+\ldots+\pi_{k-1}\right)} e^{-(\vartheta+\psi+y)\left(\Delta_{k-j+1}+\ldots+\Delta_{k-1}\right)} \\
& \quad \times E u^{x_{k}}\left(1-x^{x_{k}}\right) e^{-i \phi \pi_{k}} e^{-\vartheta \Delta_{k}}\left(1-e^{-y \Delta_{k}}\right) \\
& \quad \hat{\gamma}^{j-1}[g(1,0)-g(1, y)-g(x, 0)+g(x, y)] .
\end{aligned}
$$

$F_{j k}^{*}(\theta, x, y)$ can be copied from Case $(i)$, equation (3.30), of $\Phi_{\nu_{1}<\nu_{2}}$ as

$$
F_{j k}^{*}(\theta, x, y)=\frac{1}{\theta} \gamma_{0} \gamma^{k-j-1}[\hat{\gamma}-\hat{\gamma}(\theta)]
$$

to get

$$
\begin{aligned}
\Phi_{j k}^{*}(\theta, & x, y)=\frac{1}{\theta} \gamma_{0} \gamma^{k-j-1}[\hat{\gamma}-\hat{\gamma}(\theta)] \hat{\gamma}^{j-1} \\
& \times[g(1,0)-g(1, y)-g(x, 0)+g(x, y)]
\end{aligned}
$$

yielding

$$
\sum_{k=1}^{\infty} \sum_{j=1}^{k-1} \Phi_{j k}^{*}(\theta, x, y)=\gamma_{0} \frac{g_{12}-g_{1}-g_{2}+g}{(1-\gamma)(1-\hat{\gamma})} \Gamma \gamma
$$

Case $(i i)$. With $j=0, k>0$,

$$
\begin{aligned}
& \quad \Phi_{0 k}(t, x, y) \\
& =E z^{A_{k-1}} e^{-i \eta P_{k-1}} v^{A_{k-1}} e^{-i \varphi P_{k-1}} e^{-\psi t_{k-1}} u^{A_{k}} e^{-i \phi P_{k}} e^{-\vartheta t_{k}} \\
& \quad \times \mathbf{1}_{\left[t_{k-1}, t_{k}\right)}(t)\left(x^{A_{k-1}}-x^{A_{k}}\right)\left(e^{-y t_{k-1}}-e^{-y t_{k}}\right) \\
& \quad=\Phi_{0 k}^{(1)}(t, x, y)-\Phi_{0 k}^{(2)}(t, x, y),
\end{aligned}
$$

where

$$
\begin{aligned}
& \Phi_{0 k}^{(1)}(t, x, y)=E\left[\mathbf{1}_{\left[t_{k-1}, t_{k}\right)}(t)(u v z x)^{A_{k-1}}\right. \\
& \times e^{-i(\eta+\varphi+\phi) P_{k-1}} e^{-(\psi+\vartheta+y) t_{k-1}} \\
& \left.\times\left(u^{x_{k}} e^{-i \phi \pi_{k}} e^{-\vartheta \Delta_{k}}-(u x)^{x_{k}} e^{-i \phi \pi_{k}} e^{-\vartheta \Delta_{k}}\right)\right]
\end{aligned}
$$


and

$$
\begin{aligned}
& \Phi_{0 k}^{(2)}(t, x, y) \\
= & E\left[\mathbf{1}_{\left[t_{k-1}, t_{k}\right)}(t)(u v z x)^{A_{k-1}} e^{-i(\eta+\varphi+\phi) P_{k-1}} e^{-(\psi+\vartheta+y) t_{k-1}}\right. \\
& \left.\times\left(u^{x_{k}} e^{-i \phi \pi_{k}} e^{-(\vartheta+y) \Delta_{k}}-(u x)^{x_{k}} e^{-i \phi \pi_{k}} e^{-(\vartheta+y) \Delta_{k}}\right)\right] .
\end{aligned}
$$

Here $\Phi_{0 k}^{(2)}(t, x, y)$ is identical to that of Case $(i i)$ for $\Phi_{\nu_{1}<\nu_{2}}(t)$ implying that

$$
\begin{aligned}
& \Phi_{0 k}^{*(2)}(\theta, x, y) \\
= & \gamma_{0} \gamma^{k-1}\left[\Gamma g_{1}-\Gamma g_{1}(\theta)\right]=\frac{1}{\theta}\left[g_{1}-g-g_{1}(\theta)+g(\theta)\right],
\end{aligned}
$$

whereas

$$
\begin{aligned}
& \Phi_{0 k}^{*(1)}(t, x, y) \\
& =\sum_{a=0}^{\infty}(u v z x)^{a} \sum_{\xi=0}^{\infty}\left[u^{\xi}-(u x)^{\xi}\right] \int_{p=-\infty}^{\infty} e^{-i(\varphi+\phi+\eta) p} \\
& \times \int_{q=-\infty}^{\infty} e^{-i \phi q} \int_{s=0}^{\infty} e^{-(\vartheta+\psi+y) s} \int_{\delta=0}^{\infty} e^{-\vartheta \delta} \int_{t=s}^{s+\delta} e^{-\theta t} d t \\
& \quad \times P_{A \otimes P \otimes t_{k-j-1} \otimes x_{k-j} \otimes \pi \otimes \Delta}(a, d p, d s, \xi, d s, d \delta) \\
& =\frac{1}{\theta} \gamma_{0} \gamma^{k-1}\left[g_{12}-g_{2}-g_{12}(\theta)+g_{2}(\theta)\right] .
\end{aligned}
$$

Hence,

$$
\begin{aligned}
\Phi_{0 k}^{*}(t, x, y)= & \frac{1}{\theta} \gamma_{0} \gamma^{k-1}\left[g_{12}-g_{2}-g_{12}(\theta)\right. \\
& \left.+g_{2}(\theta)-g_{1}+g+g_{1}(\theta)-g(\theta)\right]
\end{aligned}
$$

and from (3.55),

$$
\begin{aligned}
& \sum_{k=1}^{\infty} \Phi_{0 k}^{*}(\theta, x, y)=\frac{\gamma_{0}}{1-\gamma} \frac{1}{\theta}\left[g_{12}-g_{2}-g_{12}(\theta)\right. \\
&\left.+g_{2}(\theta)-g_{1}+g+g_{1}(\theta)-g(\theta)\right]
\end{aligned}
$$

Case $(\boldsymbol{i i i}) \cdot j=k>0$. Here we have

$$
\begin{aligned}
& \Phi_{k k}(t, x, y) \\
& =E v^{A_{k-1}} e^{-i \varphi P_{k-1}} e^{-\psi t_{k-1}} u^{A_{k}} e^{-i \phi P_{k}} e^{-\vartheta t_{k}} \mathbf{1}_{\left[0, t_{0}\right)}(t) \\
& \quad \times\left(x^{A_{k-1}}-x^{A_{k}}\right)\left(e^{-y t_{k-1}}-e^{-y t_{k}}\right) \\
& =E \mathbf{1}_{\left[0, t_{0}\right)}(t)(u v x)^{A_{0}} e^{-i(\varphi+\phi) P_{0}} e^{-(\vartheta+\psi+y) t_{0}} \\
& \times E(u v x)^{x_{1}+\ldots+x_{k-1}} e^{-i(\varphi+\phi)\left(\pi_{1}+\ldots+\pi_{k-1}\right)} \\
& \times e^{-(\vartheta+\psi+y)\left(\Delta_{1}+\ldots+\Delta_{k-1}\right)} \\
& \times E\left(u^{x_{k}}-(u x)^{x_{k}}\right) e^{-i \phi \pi_{k}}\left(e^{-\vartheta \Delta_{k}}-e^{-(\vartheta+y) \Delta_{k}}\right) \\
& =F_{k k}(t, x, y) f_{k}(x, y),
\end{aligned}
$$

where

$$
F_{k k}(t, x, y)=E \mathbf{1}_{\left[0, t_{0}\right)}(t)(u v x)^{A_{0}} e^{-i(\varphi+\phi) P_{0}} e^{-(\vartheta+\psi+y) t_{0}}
$$

and

$$
\begin{aligned}
& f_{k}(x, y)=E(u v x)^{x_{1}+\ldots+x_{k-1}} \\
& \quad \times e^{-i(\varphi+\phi)\left(\pi_{1}+\ldots+\pi_{k-1}\right)} e^{-(\vartheta+\psi+y)\left(\Delta_{1}+\ldots+\Delta_{k-1}\right)} \\
& \quad \times E\left[u^{x_{k}} e^{-i \phi \pi_{k}} e^{-\vartheta \Delta_{k}}-u^{x_{k}} e^{-i \phi \pi_{k}} e^{-(\vartheta+y) \Delta_{k}}\right. \\
& \left.-(u x)^{x_{k}} e^{-i \phi \pi_{k}} e^{-(\vartheta+y) \Delta_{k}}+(u x)^{x_{k}} e^{-i \phi \pi_{k}} e^{-(\vartheta+y) \Delta_{k}}\right] \\
& =\hat{\gamma}^{k-1}[g(1,0)-g(1, y)-g(x, 0)+g(x, y)]
\end{aligned}
$$

so that

$$
F_{k k}^{*}(\theta, x, y)=\Gamma \gamma_{0} .
$$

Thus, because $\|\gamma\|<1$ and $\|\hat{\gamma}\|<1$, as pointed out in (3.34),

$$
\sum_{k=1}^{\infty} \Phi_{0 k}^{*}(\theta, x, y)=\frac{g_{12}-g_{1}-g_{2}+g}{1-\hat{\gamma}} \Gamma \gamma_{0}
$$

completing Case (iii).

Case $(i v)$. For $j=k=0$, we have

$$
\begin{gathered}
\Phi_{00}(t, x, y) \\
=E u^{A_{0}} e^{-i \phi P_{0}} e^{-\vartheta t_{0}} \mathbf{1}_{\left[0, t_{0}\right)}(t)\left(1-x^{A_{0}}\right)\left(1-e^{-y t_{0}}\right) \\
=E e^{-i \phi P_{0}} \mathbf{1}_{\left[0, t_{0}\right)}(t)\left(u^{A_{0}}-(u x)^{A_{0}}\right)\left(e^{-\vartheta t_{0}}-e^{-(\vartheta+y) t_{0}}\right) \\
=E \mathbf{1}_{\left[0, t_{0}\right)}(t)\left[u^{A_{0}} e^{-i \phi P_{0}} e^{-\vartheta t_{0}}-(u x)^{A_{0}} e^{-i \phi P_{0}} e^{-\vartheta t_{0}}\right. \\
\left.\quad+u^{A_{0}} e^{-i \phi P_{0}} e^{-(\vartheta+y) t_{0}}-(u x)^{A_{0}} e^{-i \phi P_{0}} e^{-(\vartheta+y) t_{0}}\right]
\end{gathered}
$$

and

$$
\Phi_{00}^{*}(\theta, x, y)=\Phi_{00}^{*(1)}(\theta, x, y)-\Phi_{00}^{*(2)}(\theta, x, y)
$$

with $\Phi_{00}^{*(2)}(\theta, x, y)$ exactly the same as of Case $(i v)$ under $\Phi_{\nu_{2}<\nu_{1}}$, that is,

$$
\Phi_{00}^{*(2)}(\theta, x, y)=\frac{1}{\theta}\left[g_{01}-g_{0}-g_{01}(\theta)+g_{0}(\theta)\right] .
$$

Unlike $\Phi_{00}^{(2)}(t, x, y)$, the functional $\Phi_{00}^{(1)}(t, x, y)$ has $y=0$ in its third component. Thus

$$
\Phi_{00}^{*(1)}(\theta, x, y)=\frac{1}{\theta}\left[g_{012}-g_{02}-g_{012}(\theta)+g_{02}(\theta)\right]
$$

implying that

$$
\begin{aligned}
& \Phi_{00}^{*}(\theta, x, y)= \Phi_{00}^{*(1)}(\theta, x, y)-\Phi_{00}^{*(2)}(\theta, x, y) \\
&=\frac{1}{\theta}\left[g_{012}-g_{02}-g_{012}(\theta)\right. \\
&\left.+g_{02}(\theta)-g_{01}+g_{0}+g_{01}(\theta)-g_{0}(\theta)\right]
\end{aligned}
$$


completing Case $(i v)$.

Now summing up $\Phi^{*}$ 's from all four cases, under the provisions of (3.34), gives

$$
\begin{aligned}
& \Phi_{\nu_{1}=\nu_{2}}^{*}(\theta, x, y) \\
& =\gamma_{0} \frac{g_{12}-g_{1}-g_{2}+g}{(1-\gamma)(1-\hat{\gamma})} \Gamma \gamma+\frac{g_{12}-g_{1}-g_{2}+g}{1-\hat{\gamma}} \Gamma \gamma_{0} \\
& \quad+\frac{\gamma_{0}}{1-\gamma} \frac{1}{\theta}\left[g_{12}-g_{2}-g_{12}(\theta)+g_{2}(\theta)\right. \\
& \left.\quad-g_{1}+g+g_{1}(\theta)-g(\theta)\right] \\
& \quad+\frac{1}{\theta}\left[g_{012}-g_{02}-g_{012}(\theta)\right. \\
& \left.\quad+g_{02}(\theta)-g_{01}+g_{0}+g_{01}(\theta)-g_{0}(\theta)\right] .
\end{aligned}
$$

Finally, the transformation of the main functional $\Phi_{\nu}$ reads

$$
\begin{gathered}
\hat{\Phi}^{*}(\theta, x, y)=\int_{t=0}^{\infty} e^{-\theta t} D_{p} \mathcal{L C} \mathcal{C}_{q}\left[E z^{A(t)} e^{-i \eta P(t)} v^{A_{\nu-1}}\right. \\
\quad \times e^{-i \varphi P_{\nu-1}} e^{-\psi t_{\nu-1}} u^{A_{\nu}} e^{-i \phi P_{\nu}} e^{-\vartheta} \\
=\hat{\Phi}_{\nu_{1}<\nu_{2}}^{*}(\theta, x, y)+\hat{\Phi}_{\nu_{1}>\nu_{2}}^{*}(\theta, x, y)+\hat{\Phi}_{\nu_{1}=\nu_{2}}^{*}(\theta, x, y) \\
=\frac{\gamma_{0}}{(1-\gamma)(1-\hat{\gamma})} \Gamma \gamma \Gamma g_{12} \theta+\frac{\gamma_{0}}{1-\gamma}\left[\Gamma g_{12}-\Gamma g_{12}(\theta)\right] \\
\quad+\frac{\Gamma \gamma_{0}}{1-\hat{\gamma}} \Gamma g_{12} \theta+\Gamma g_{012}-\Gamma g_{012}(\theta)
\end{gathered}
$$

The above result can be summarized in the following theorem.

Theorem 3.1. In a stochastic game of two active hostile players $A$ and $B$ (where $B$ is the point process $\left\{t_{0}, t_{1}, \ldots\right\}$ ) and one passive player $C$, the transform

$$
\begin{aligned}
\hat{\Phi}^{*}(\theta, x, y) \\
=\int_{t=0}^{\infty} e^{-\theta t} D_{p} \mathcal{L} \mathcal{C}_{q}\left[E z^{A(t)} e^{-i \eta P(t)} v^{A_{\nu-1}} e^{-i \varphi P_{\nu-1}} e^{-\psi t_{\nu-1}}\right. \\
\left.\times u^{A_{\nu}} e^{-i \phi P_{\nu}} e^{-\vartheta t_{\nu}} \mathbf{1}_{\left[0, t_{\nu}\right)}(t)\right](x, y) d t
\end{aligned}
$$

of the joint functional $\Phi_{\nu}$ (1.1) describing the evolution of the game at any time $t$ from its inception to the end at $t_{\nu}$ (the first passage time) satisfies the following formulas:

$$
\begin{aligned}
\hat{\Phi}^{*}(\theta, x, y)= & \frac{\gamma_{0}}{(1-\gamma)(1-\hat{\gamma})} \Gamma \gamma \Gamma g_{12} \theta \\
& +\frac{\gamma_{0}}{1-\gamma}\left[\Gamma g_{12}-\Gamma g_{12}(\theta)\right] \\
& +\frac{\Gamma \gamma_{0}}{1-\hat{\gamma}} \Gamma g_{12} \theta+\Gamma g_{012}-\Gamma g_{012}(\theta) .
\end{aligned}
$$

where

$$
\begin{aligned}
\Gamma \gamma=\frac{1}{\theta}[\gamma(u v x, \varphi+\phi, \vartheta+\psi+y) & \\
& -\gamma(u v x, \varphi+\phi, \theta+\vartheta+\psi+y)],
\end{aligned}
$$

$$
\begin{aligned}
& \Gamma g_{12}(\theta)=\frac{1}{\theta}\left[g_{12}(\theta)-g(\theta)\right] \\
& =\frac{1}{\theta}[\gamma(u, \phi, \theta+\vartheta+y)-\gamma(u x, \phi, \theta+\vartheta+y)], \\
& \Gamma g_{12}=\frac{1}{\theta}\left[g_{12}-g\right]=\frac{1}{\theta}[\gamma(u, \phi, \vartheta)-\gamma(u x, \phi, \vartheta+y)], \\
& \Gamma g_{012}(\theta)=\frac{1}{\theta}\left[g_{012}(\theta)-g(\theta)\right] \\
& =\frac{1}{\theta}\left[\gamma_{0}(u, \phi, \theta+\vartheta+y)-\gamma_{0}(u x, \phi, \theta+\vartheta+y)\right], \\
& \Gamma g_{012}=\frac{1}{\theta}\left[g_{012}-g\right] \\
& =\frac{1}{\theta}\left[\gamma_{0}(u, \phi, \vartheta+y)-\gamma_{0}(u x, \phi, \vartheta+y)\right], \\
& \hat{\gamma}=\gamma(u v x, \varphi+\phi, \vartheta+\psi+y), \\
& \gamma=\gamma(u v z x, \varphi+\phi+\eta, \theta+\vartheta+\psi+y), \\
& \gamma_{0}=\gamma_{0}(u v z x, \varphi+\phi+\eta, \theta+\vartheta+\psi+y) \text {. }
\end{aligned}
$$

Theorem 3.1 provides us with a closed-form expression in terms of the triple transform $\hat{\Phi}^{*}$ of functional $\Phi_{\nu}$. The inversion of $\Phi^{\hat{*}}$ is our main objective of the next section that we combine with applications.

\section{Applications to Option Trading}

For an illustration, consider the following special cases pertaining to a constantly fluctuating stock price of some company over the times $t_{0}=0, t_{1}, t_{2}, \ldots$ that starts off at time zero with a price of $\pi_{0}$ which is assumed to be a.s. a constant r.v. equal to $p_{0}$. Without making any further mention, we always refer to Theorem 3.1 under special assumptions.

\section{Case 1. Observation of process $\boldsymbol{P}(\boldsymbol{t})$ when Player A's threshold is $M=1$.}

1a. Suppose we are interested in the characteristics of the process around the period when the stock price drops for the first time or when maturity $T$ is reached, whichever comes first. Because the stock prices cannot be modeled by a monotone process, we regard the observed prices upon $t_{k}$ 's as forming the passive component, and introduce the active component

$$
X_{n}=\left\{\begin{array}{ll}
0, & \pi_{n} \geq 0 \\
1, & \pi_{n}<0
\end{array} .\right.
$$

Our next assumption is that the marginal distribution of $\pi_{1}$ is Laplace with parameter $\mu$ and zero shif 1 Thus the pdf of $\pi_{1}$ is

$$
f_{\pi_{1}}(x)=\frac{1}{2} \mu e^{-\mu|x|}, x \in \mathbb{R} .
$$

Also, suppose $\Delta_{n} \sim \operatorname{Exp}(\lambda), n=1,2,3 \ldots$ Then, with

$$
\gamma_{0}(u, \phi, \vartheta)=E u^{A_{0}} e^{-i \phi P_{0}} e^{-\vartheta t_{0}}=e^{-i \phi p_{0}}
$$

\footnotetext{
${ }^{1}$ We note that this assumption is impractical, because it can send a stock to a negative territory. However, we do it for illustration purposes only. A more reasonable assumption would be a Laplace distribution with a non-zero shift.
} 
under $P_{0}=\pi_{0}=p_{0}$ a.s. constant, and $A_{0}=t_{0}=0$ a.s., we have

$$
\gamma_{0}=\gamma_{0}(u v z x, \varphi+\phi+\eta, \theta+\vartheta+\psi+y)=e^{-i(\varphi+\phi+\eta) p_{0}}
$$

and

$$
\Gamma \gamma_{0}=\Gamma g_{012}=\Gamma g_{012}(\theta)=0
$$

Hence,

$$
\begin{aligned}
\Phi^{*}(\theta, x, y)= & \frac{e^{-i(\varphi+\phi+\eta) p_{0}}}{(1-\gamma)(1-\hat{\gamma})} \Gamma \gamma \Gamma g_{12} \theta \\
& +\frac{e^{-i(\varphi+\phi+\eta) p_{0}}}{1-\gamma}\left[\Gamma g_{12}-\Gamma g_{12}(\theta)\right]
\end{aligned}
$$

\section{implying that}

$$
\begin{gathered}
e^{i(\varphi+\phi+\eta) p_{0}} \theta \Phi^{*}(\theta, x, y) \\
=\int_{t=0}^{\infty} e^{-\theta t} \mathcal{D}_{p} \mathcal{L} \mathcal{C}_{q}\left(E \left[z^{A(t)} e^{-i \eta P(t)} v^{A_{\nu-1}} e^{-i \varphi P_{\nu-1}}\right.\right. \\
\left.\left.\times e^{-\psi t_{\nu-1}} u^{A_{\nu}} e^{-i \phi P_{\nu}} e^{-\vartheta t_{\nu}} \mathbf{1}_{\left[0, t_{\nu}\right)}(t)\right]_{\nu_{1}(p), \nu_{2}(q)}\right)(x, y) d t \\
=[[1-\gamma(u v z x, \varphi+\phi+\eta, \theta+\vartheta+\psi+y)] \\
\quad \times[1-\gamma(u v x, \varphi+\phi, \vartheta+\psi+y)]]^{-1} \\
\times[\gamma(u v x, \varphi+\phi, \vartheta+\psi+y) \\
-\gamma(u v x, \varphi+\phi, \theta+\vartheta+\psi+y)] \\
\times[\gamma(u, \phi, \vartheta)-\gamma(u x, \phi, \vartheta+y)] \\
+[1-\gamma(u v z x, \varphi+\phi+\eta, \theta+\vartheta+\psi+y)] \\
\times[\gamma(u, \phi, \vartheta)-\gamma(u x, \phi, \vartheta+y)-\gamma(u, \phi, \theta+\vartheta) \\
+\gamma(u x, \phi, \theta+\vartheta+y)] . \quad(4.6)
\end{gathered}
$$

Next, with $z=v=u=1$,

$$
\begin{aligned}
e^{i(\varphi+\phi+\eta) p_{0}} & \theta \Phi^{*}(\theta, x, y)=e^{i(\varphi+\phi+\eta) p_{0}} \theta \\
\times & \int_{t=0}^{\infty} e^{-\theta t} D_{p} \mathcal{L} \mathcal{C}_{q}\left[E e^{-i \eta P(t)} e^{-i \varphi P_{\nu-1}}\right. \\
\times & \left.e^{-\psi t_{\nu-1}} e^{-i \phi P_{\nu}} e^{-\vartheta t_{\nu}} \mathbf{1}_{\left[0, t_{\nu}\right)}(t)\right](x, y) d t \\
= & {[[1-\gamma(x, \varphi+\phi+\eta, \theta+\vartheta+\psi+y)]} \\
& \times[1-\gamma(x, \varphi+\phi, \vartheta+\psi+y)]]^{-1}
\end{aligned}
$$

$\times[\gamma(x, \varphi+\phi, \vartheta+\psi+y)-\gamma(x, \varphi+\phi, \theta+\vartheta+\psi+y)]$$$
\times[\gamma(1, \phi, \vartheta)-\gamma(x, \phi, \vartheta+y)]
$$$$
+[1-\gamma(x, \varphi+\phi+\eta, \theta+\vartheta+\psi+y)]^{-1}
$$$$
\times[\gamma(1, \phi, \vartheta)-\gamma(x, \phi, \vartheta+y)
$$$$
-\gamma(1, \phi, \theta+\vartheta)+\gamma(x, \phi, \theta+\vartheta+y)]
$$

Furthermore, with $\varphi=\psi=\phi=\vartheta=0$,

$$
\begin{gathered}
e^{i \eta p_{0}} \theta \Phi^{*}(\theta, x, y) \\
=e^{i \eta p_{0}} \theta \int_{t=0}^{\infty} e^{-\theta t} D_{p} \mathcal{L C} \mathcal{C}_{q}\left[E e^{-i \eta P(t)} \mathbf{1}_{\left[0, t_{\nu}\right)}(t)\right](x, y) d t \\
=[[1-\gamma(x, \eta, \theta+y)][1-\gamma(x, 0, y)]]^{-1} \\
\frac{1}{\theta}[\gamma(x, 0, y)-\gamma(x, 0, \theta+y)] \\
\times[\gamma(1,0,0)-\gamma(x, 0, y)] \\
+[1-\gamma(x, \eta, \theta+y)]^{-1} \frac{1}{\theta}[\gamma(1,0,0)-\gamma(x, 0, y) \\
-\gamma(1,0, \theta)+\gamma(x, 0, \theta+y)]
\end{gathered}
$$

\section{Assuming position independent marking,}

$\gamma(u, \phi, \vartheta)=E u^{x_{1}} e^{-i \phi \pi_{1}} e^{-\vartheta \Delta_{1}}=E u^{x_{1}} e^{-i \phi \pi_{1}} \cdot E e^{-\vartheta \Delta_{1}}$, where $E e^{-\vartheta \Delta_{1}}=\frac{\lambda}{\lambda+\vartheta}$, as $\Delta_{1} \sim \operatorname{Exp}(\lambda)$. Then, under $M=1$,

$$
\begin{gathered}
\mathcal{D}_{x}^{0} e^{i \eta p_{0}} \theta \Phi^{*}(\theta, x, y)=e^{i \eta p_{0}} \theta \Phi^{*}(\theta, 0, y) \\
=[[1-\gamma(0, \eta, \theta+y)][1-\gamma(0,0, y)]]^{-1} \\
\times[\gamma(0,0, y)-\gamma(0,0, \theta+y)][\gamma(1,0,0)-\gamma(0,0, y)] \\
+[1-\gamma(0, \eta, \theta+y)]^{-1}[\gamma(1,0,0)-\gamma(0,0, y) \\
-\gamma(1,0, \theta)+\gamma(0,0, \theta+y)]
\end{gathered}
$$

Now,

$$
\begin{aligned}
\gamma(0, \phi, \vartheta)=E u^{x_{1}} e^{-i \phi \pi_{1}} & \left.\right|_{u=0} E e^{-\Delta_{1} \vartheta} \\
& =E \mathbf{1}_{\left\{\pi_{1} \geq 0\right\}} e^{-i \phi \pi_{1}} \frac{\lambda}{\lambda+\vartheta} .
\end{aligned}
$$

Due to our assumption on the pdf of $\pi_{1}$,

$$
\begin{aligned}
& \gamma(0, \phi)=E \mathbf{1}_{\left\{\pi_{1} \geq 0\right\}} e^{-i \phi \pi_{1}} \\
= & \int_{x=0}^{\infty} e^{-i \phi x} \frac{1}{2} \mu e^{-\mu x} d x=\frac{1}{2} \frac{\mu}{\mu+i \phi} ; \gamma(0,0)=\frac{1}{2} .
\end{aligned}
$$
have

Because $E e^{-i \phi \pi_{1}}=E e^{-i \phi \pi_{1}}\left(\mathbf{1}_{\left\{\pi_{1} \geq 0\right\}}+\mathbf{1}_{\left\{\pi_{1}<0\right\}}\right)$, we

$$
\begin{aligned}
E e^{-i \phi \pi_{1}} & =\left.E u^{x_{1}} e^{-i \phi \pi_{1}}\right|_{u=1} E e_{\vartheta=0}^{-\Delta_{1} \vartheta}=\gamma(1, \phi, 0) \\
& =\frac{1}{2} \frac{\mu}{\mu+i \phi}+\int_{x=-\infty}^{0} e^{-i \phi x} \frac{1}{2} \mu e^{\mu x} d x=\frac{\mu^{2}}{\mu^{2}+\phi^{2}}
\end{aligned}
$$

Thus,

$$
\begin{aligned}
\gamma(1, \phi, \vartheta)=E e^{-i \phi \pi_{1}} E e^{-\Delta_{1} \vartheta} & =E e^{-i \phi \pi_{1}} \frac{\lambda}{\lambda+\vartheta} \\
& =\frac{\mu^{2}}{\mu^{2}+\phi^{2}} \frac{\lambda}{\lambda+\vartheta}
\end{aligned}
$$


and

$$
\gamma(1,0, \vartheta)=\frac{\lambda}{\lambda+\vartheta}
$$

implying that

$$
\begin{aligned}
& e^{i \eta p_{0}} \Phi^{*}(\theta, 0, y) \\
& =e^{i \eta p_{0}} \int_{t=0}^{\infty} e^{-\theta t} D_{p} \mathcal{L} \mathcal{C}_{q}\left[E e^{-i \eta P(t)} \mathbf{1}_{\left[0, t_{\nu}\right)}(t)\right](x, y) d t \\
& =\left[1-\frac{1}{2} \frac{\mu}{\mu+i \eta} \frac{\lambda}{\lambda+\theta+y}\right]^{-1} \frac{1}{\lambda+\theta} .
\end{aligned}
$$

With the notation

$$
A=\frac{1}{2} \frac{\lambda \mu}{\mu+i \eta} \text { and } B=\lambda-A,
$$

we have (4.15) in the form

$$
e^{i \eta p_{0}} \Phi^{*}(\theta, 0, y)=\frac{1}{\lambda+\theta}+A \frac{1}{\lambda+\theta} \frac{1}{\theta+y+B} .
$$

Due to the relation between the Laplace-Carson and Laplace transforms we will seek the Laplace inverse of the function

$$
F(y, \theta)=\frac{1}{\lambda+\theta} \frac{1}{y}+A \frac{1}{\lambda+\theta} \frac{1}{\theta+y+B} \frac{1}{y}
$$

and then its Laplace inverse with respect to $\theta$ or other way around which is about the same complexity.

With

$$
\begin{aligned}
\mathcal{L}_{y}^{-1}[F(y, \theta)](T)= & \frac{1}{\lambda+\theta}+A \frac{1}{\lambda+\theta} \frac{1}{B+\theta} \\
& -A \frac{1}{\lambda+\theta} \frac{1}{B+\theta} e^{-B T} e^{-\theta T}
\end{aligned}
$$

and

$$
\begin{aligned}
& \mathcal{L}_{\theta}^{-1}\left\{\mathcal{L}_{y}^{-1}[F(y, \theta)](T)\right\}(t) \\
&= \mathcal{L}_{\theta}^{-1}\left(\frac{1}{\lambda+\theta}\right)(t)+A \mathcal{L}_{\theta}^{-1}\left(\frac{1}{\lambda+\theta} \frac{1}{B+\theta}\right)(t) \\
& \quad-A e^{-B T} \mathcal{L}_{\theta}^{-1}\left(\frac{1}{\lambda+\theta} \frac{1}{B+\theta} e^{-\theta T}\right)(t) \\
&=e^{-\lambda t}+\frac{A}{B-\lambda} e^{-\lambda t}\left[1-\mathbf{1}_{(T, \infty)}(t) e^{(\lambda-B) T}\right] \\
& \quad-\frac{A}{B-\lambda} e^{-B t}\left[1-\mathbf{1}_{(T, \infty)}(t)\right],
\end{aligned}
$$

we arrive at

$$
\begin{aligned}
e^{i \eta p_{0}} \mathcal{L}_{\theta}^{-1} \mathcal{L}_{y}^{-1} \frac{1}{y} \Phi^{*}(\theta, 0, y) & (t, T) \\
=e^{-\lambda t}+\frac{A}{B-\lambda} e^{-\lambda t} & {\left[1-\mathbf{1}_{(T, \infty)}(t) e^{(\lambda-B) T}\right] } \\
& -\frac{A}{B-\lambda} e^{-B t} \mathbf{1}_{[0, T]}(t)
\end{aligned}
$$

and

$$
\begin{aligned}
& E e^{-i \eta P(t)} \mathbf{1}_{\left[0, t_{\nu}\right)}(t) \\
& =e^{-i \eta p_{0}}\left[e^{-\lambda t}+\frac{A}{B-\lambda} e^{-\lambda t}\left[1-\mathbf{1}_{(T, \infty)}(t) e^{(\lambda-B) T}\right]\right. \\
& \left.-\frac{A}{B-\lambda} e^{-B t} \mathbf{1}_{[0, T]}(t)\right], \quad \text { (4.2) }
\end{aligned}
$$

where (4.16) implies that $\frac{A}{B-\lambda}=-1$. So

$$
\begin{aligned}
& E e^{-i \eta P(t)} \mathbf{1}_{\left[0, t_{\nu}\right)}(t) \\
& \quad=e^{-\lambda t} e^{-i \eta p_{0}}\left[e^{A t} \mathbf{1}_{[0, T]}(t)+e^{A T} \mathbf{1}_{(T, \infty)}(t)\right] .
\end{aligned}
$$

In particular,

$$
\begin{aligned}
E \mathbf{1}_{\left[0, t_{\nu}\right)}(t) & =P\left\{t_{\nu}>t\right\} \\
= & e^{-\lambda t}\left[e^{\lambda t / 2} \mathbf{1}_{[0, T]}(t)+e^{\lambda T / 2} \mathbf{1}_{(T, \infty)}(t)\right] .
\end{aligned}
$$

This predicted result $P\left\{t_{\nu}>t\right\}$ in (4.24) is simple to compute given the parameters $\lambda$ and $T$.

Monte Carlo Simulation. To test the prediction, we implemented a Monte Carlo method to generate a sample from the distribution of $t_{\nu}$ in MATLAB by simulating $n$ paths of the process in this special case. For each path, we use the following procedure.

1. Generate random numbers $x_{1}=\mathbf{1}_{(0, \infty)}\left(p_{1}\right), x_{2}=$ $\mathbf{1}_{(0, \infty)}\left(p_{2}\right), \ldots$ where each $p_{i}$ is from the Laplace pdf $f_{\pi_{1}}$ until their sum is $M$ and store $a_{j}=\sum_{i=1}^{j} x_{i}$ for $j=1, \ldots, v_{1}$ where $v_{1}=\min \left\{n: a_{n}>M\right\}$.

2. Generate random numbers $d_{1}, d_{2}, \ldots$ from the $\operatorname{Exp}(\lambda)$ distribution until their sum is $T$ and store $\tau_{j}=\sum_{i=1}^{j} d_{i}$ for $j=1, \ldots, v_{2}$ where $v_{2}=\min \left\{n: \tau_{n}>T\right\}$.

Then, $\tau_{v}^{i}=\tau_{\min \left\{v_{1}, v_{2}\right\}}$ is the $i$ th sample value from the distribution of $t_{\nu}$. We use these sample values to compute an empirical cumulative distribution function to approximate the true function as

$$
P\left\{t_{\nu} \leq t\right\} \approx \frac{\sum_{i=1}^{n} \mathbf{1}_{\left[\tau_{v}^{i}, \infty\right)}(t)}{n}
$$

for each $t$ in a mesh from 0 to $\max \left\{\tau_{v}^{i}: i=1, \ldots, n\right\}$, and assume $P\left\{t_{\nu} \leq t\right\} \approx 1$ for later times $t$.

In Figure 1 below, we plot the predicted cumulative distribution function $P\left\{t_{\nu} \leq t\right\}=1-P\left\{t_{\nu}>t\right\}$ using (4.24) under the special case where $\mu=1, \lambda=1, T=10$, and $M=1$ for $0 \leq t \leq 15$ (in black). In blue, we use the Monte Carlo estimate of $P\left\{t_{\nu} \leq t\right\}$ with the same parameter values on the mesh $\{0,0.01, \ldots, 15\}$ with sample size $n=1000$. 


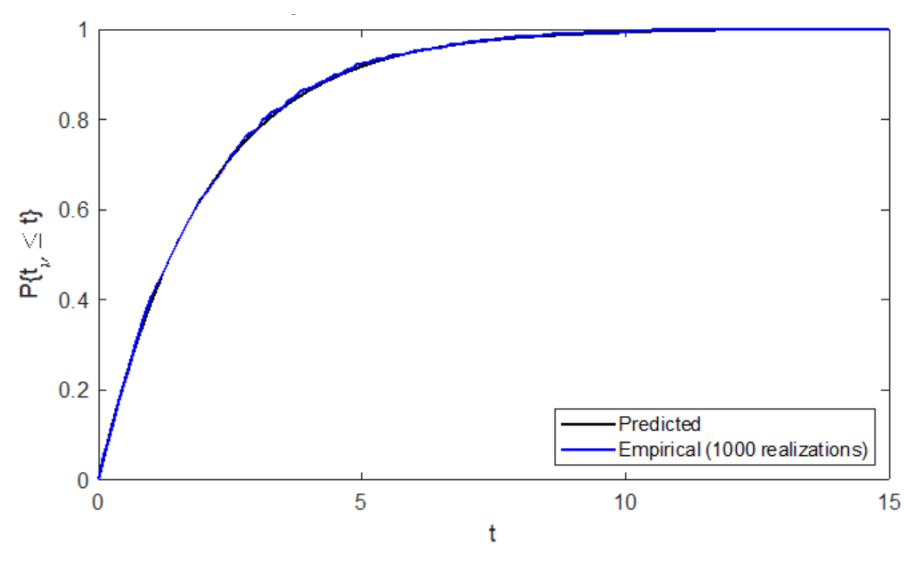

Figure 1. Predicted and empirical distribution function $P\left(t_{\nu} \leq t\right)$ in a special case where $\mu=1, \lambda=1, T=10, p_{0}=4$, and $M=1$.

Returning to (4.23),

$$
\begin{aligned}
& E e^{-i \eta P(t)} \mathbf{1}_{\left[0, t_{\nu}\right)}(t) \\
& \quad=e^{-\lambda t}\left[g(t) \mathbf{1}_{[0, T]}(t)+g(T) \mathbf{1}_{(T, \infty)}(t)\right],
\end{aligned}
$$

where

$$
g(\eta, t)=e^{\frac{1}{2} \frac{\lambda \mu}{\mu+i \eta} t-i \eta p_{0}} .
$$

Hence we have that,

$$
\frac{\partial}{\partial \eta} g(\eta, t)=g(\eta, t)\left[-i \frac{1}{2} \lambda \mu t \frac{1}{(\mu+i \eta)^{2}}-i p_{0}\right]
$$

and

$$
i \frac{\partial}{\partial \eta} g(\eta, t)_{\eta=0}=e^{\frac{1}{2} \lambda t}\left(\frac{1}{2 \mu} \lambda t+p_{0}\right)
$$

implying that the expected stock price at any time $t$ confined on the interval $\left[0, t_{\nu}\right)$ is

$$
\begin{gathered}
E P(t) \mathbf{1}_{\left[0, t_{\nu}\right)}(t)=i \frac{\partial}{\partial \eta} E e^{-i \eta P(t)} \mathbf{1}_{\left[0, t_{\nu}\right)}(t)_{\eta=0} \\
=e^{-\lambda t}\left[e^{\frac{1}{2} \lambda t}\left(\frac{1}{2 \mu} \lambda t+p_{0}\right) \mathbf{1}_{[0, T]}(t)\right. \\
\left.+e^{\frac{1}{2} \lambda T}\left(\frac{1}{2 \mu} \lambda T+p_{0}\right) \mathbf{1}_{(T, \infty)}(t)\right] .
\end{gathered}
$$

The figures below compare predicted and empirical values of $E P(t) \mathbf{1}_{\left[0, t_{\nu}\right)}(t)$. Again, we simulated the process 1000 times and computed empirical means at each $t$ restricted to $\left[0, \tau_{\nu}\right)$, i.e. $E P(t) \mathbf{1}_{\left[0, t_{\nu}\right)}(t)$, via a Monte Carlo method similar to the one described above with MATLAB. Fig. 2 and Fig. 3 show the predicted values from (4.29) and empirical values agree to very high precision in two examples.

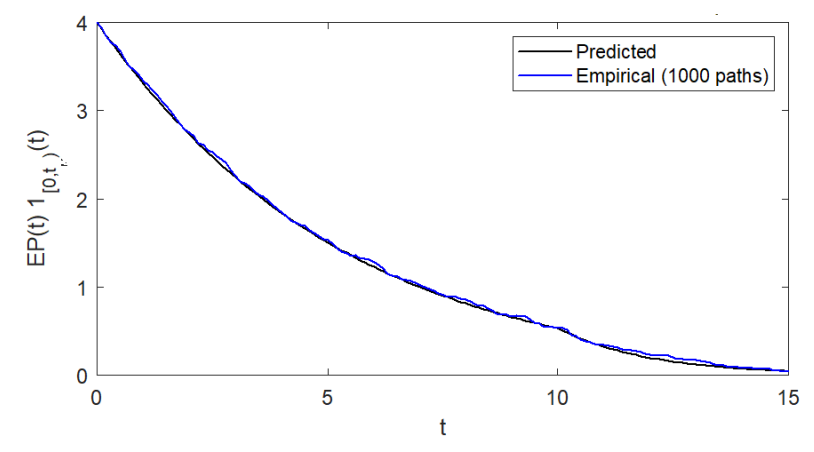

Figure 2. Predicted and empirical values of $\operatorname{EP}(t) \mathbf{1}_{\left[0, t_{\nu}\right)}(t)$ in a special case where $\mu=1, \lambda=0.5, T=10, p_{0}=4$, and $M=1$.

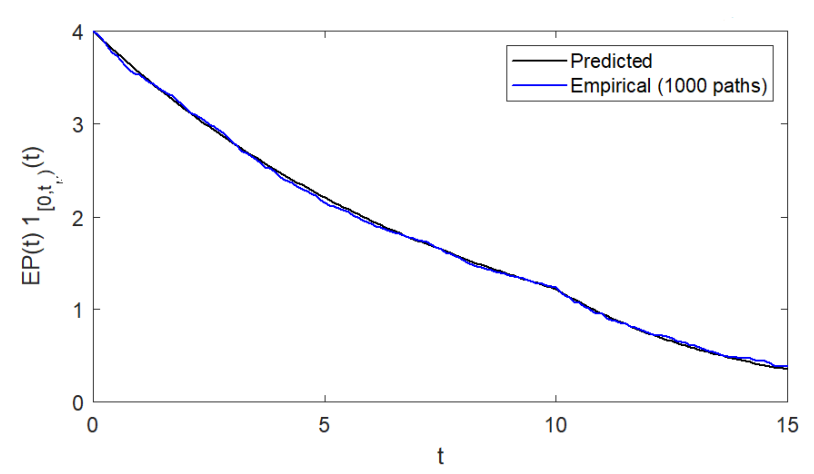

Figure 3. Predicted and empirical values of $\operatorname{EP}(t) \mathbf{1}_{\left[0, t_{\nu}\right)}(t)$ in a special case where $\mu=5, \lambda=0.25, T=10, p_{0}=4$, and $M=1$.

The following gives the joint density of the stock value at time $t \in\left[0, t_{\nu}\right)$.

\section{Proposition 1. It holds true that}

$$
\begin{aligned}
\frac{\partial}{\partial \vartheta} P\{P(t) & \left.\leq \vartheta, t_{\nu}>t\right\} \\
=e^{-\lambda t} & \left\{\mathcal{F}^{-1}\left[e^{\frac{1}{2} \lambda \frac{\mu}{\mu+i \eta} t-i \eta p_{0}}\right](\vartheta) \mathbf{1}_{[0, T]}(t)\right. \\
& \left.+\mathcal{F}^{-1}\left[e^{\frac{1}{2} \lambda \frac{\mu}{\mu+i \eta} t-i \eta p_{0}}\right](\vartheta)_{t=T} \mathbf{1}_{(T, \infty)}(t)\right\}
\end{aligned}
$$


where $\mathcal{F}^{-1}$ is the inverse of the Fourier transform, with

$$
\begin{aligned}
& \mathcal{F}^{-1}\left[e^{\frac{1}{2} \lambda \frac{\mu}{\mu+i \eta} t-i \eta p_{0}}\right](\vartheta)=\delta\left(\vartheta-p_{0}\right) \\
&+\mathbf{1}_{\mathbb{R}_{+}}\left(\vartheta-p_{0}\right) e^{-\mu\left(\vartheta-p_{0}\right)} \sum_{n=1}^{\infty} \frac{1}{n !} \frac{1}{(n-1) !} \\
& \times\left(\frac{1}{2} \lambda \mu t\right)^{n}\left(\vartheta-p_{0}\right)^{n-1} \\
&=\delta\left(\vartheta-p_{0}\right)+\mathbf{1}_{\mathbb{R}_{+}}\left(\vartheta-p_{0}\right) \frac{e^{-\mu\left(\vartheta-p_{0}\right)}}{\vartheta-p_{0}} \sum_{n=1}^{\infty} \frac{1}{n !} \frac{1}{(n-1) !} \\
& \times\left(\frac{1}{2} \lambda \mu t\left(\vartheta-p_{0}\right)\right)^{n} \\
&=\delta(\vartheta-\left.p_{0}\right)+e^{-\mu\left(\vartheta-p_{0}\right)} \sqrt{\frac{\lambda \mu t}{2\left(\vartheta-p_{0}\right)}} \\
& \times I_{1}\left(\sqrt{2 \lambda \mu t\left(\vartheta-p_{0}\right)}\right) \mathbf{1}_{\mathbb{R}_{+}}\left(\vartheta-p_{0}\right),
\end{aligned}
$$

$\delta$ is the Dirac Delta function, and $I_{1}$ is the modified Bessel function of the first kind.

The figures 4 and 5 below depict the obtained density in Proposition 1 for different values of parameter $\mu$ (of the assumed Laplace distribution) and $\lambda$ (for the exponential distribution). Akin to rate parameter effects on Laplace and exponential density amplitudes, $\mu$ appears to affect the amplitude of the density curve of Proposition 1 and to a lesser extent its shift to the right, whereas $\lambda$ only affects its amplitude.

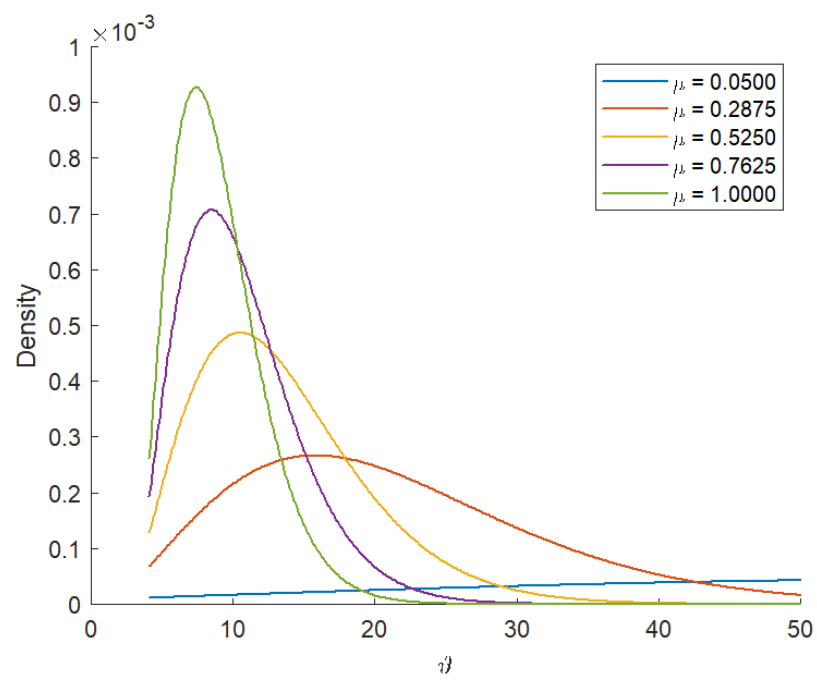

Figure 4. Joint probability density functions of $P(t)$ and $t_{\nu}$ for a special case where $\lambda=1, t=10, T=10$, and $p_{0}=4$, plotted for several values of $\mu$.

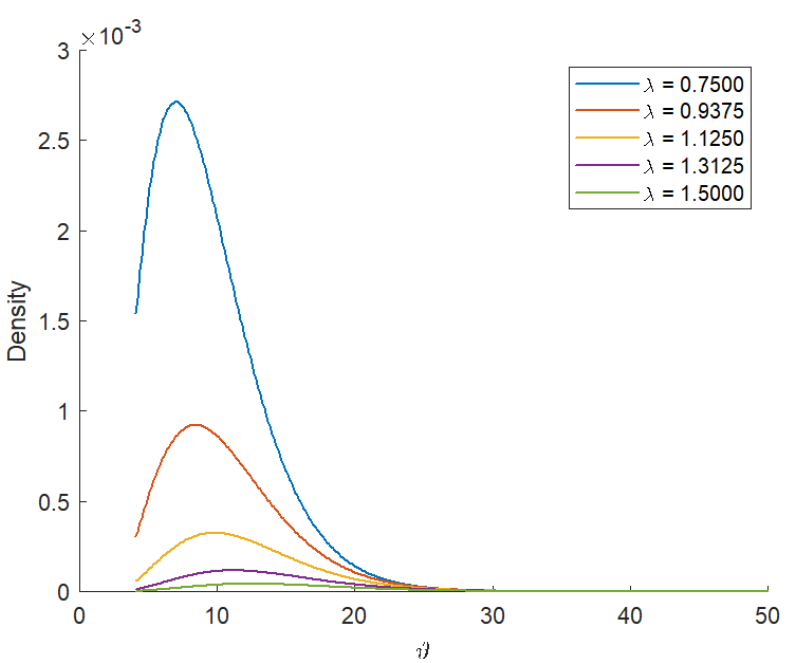

Figure 5. Joint probability density functions of $P(t)$ and $t_{\nu}$ for a special case where $\mu=0.7, t=10, T=10$, and $p_{0}=4$, plotted for several values of $\lambda$.

Proof. Recall that

$$
\mathcal{F}^{-1}[g(\eta, t)]=\frac{1}{2 \pi} \mathcal{F}[g(-\eta, t)]
$$

where $g(\eta, t)=e^{\frac{1}{2} \lambda \frac{\mu}{\mu+i \eta} t-i \eta p_{0}}$. From the expansion of

$$
e^{\frac{1}{2} \lambda t \mu \frac{1}{\mu-i \eta}}=\sum_{n=0}^{\infty} \frac{1}{n !}\left(\frac{1}{2} \lambda \mu t\right)^{n} \frac{1}{(\mu-i \eta)^{n}}
$$

and due to

$$
\begin{aligned}
\mathcal{F} & \left(\frac{1}{(\mu-i \eta)^{n}}\right)(\vartheta) \\
& =\left\{\begin{array}{cc}
0, & \vartheta<0 \\
\frac{1}{(n-1) !} 2 \pi \vartheta^{n-1} e^{-\mu \vartheta}, & \vartheta>0
\end{array}, n=1,2, \ldots,\right.
\end{aligned}
$$

and

$$
\mathcal{F}(\mathbf{1})(\vartheta)=2 \pi \delta(\vartheta)
$$

we have that

$$
\begin{aligned}
\mathcal{F}\left(e^{\frac{1}{2} \lambda t \mu \frac{1}{\mu-i \eta}}\right)(\vartheta) & \\
=2 \pi \delta(\vartheta)+2 \pi e^{-\mu \vartheta} \sum_{n=1}^{\infty} \frac{1}{n !} & \frac{1}{(n-1) !}\left(\frac{1}{2} \lambda \mu t\right)^{n} \\
& \times \vartheta^{n-1} \mathbf{1}_{\mathbb{R}_{+}}(\vartheta)
\end{aligned}
$$

From

$$
\mathcal{F}\left(g(\eta) e^{i b \eta}\right)(\vartheta)=\mathcal{F}(g(\eta))(\vartheta-b)
$$

and

$$
\begin{aligned}
\mathcal{F} g(-\eta, t)(\vartheta)=\mathcal{F} & \left(e^{\frac{1}{2} \lambda \frac{\mu}{\mu-i \eta} t+i \eta p_{0}}\right) \\
= & \mathcal{F}\left(e^{\frac{1}{2} \lambda t \mu \frac{1}{\mu-i \eta}}\right)\left(\vartheta-p_{0}\right)=2 \pi \delta\left(\vartheta-p_{0}\right) \\
\left.+2 \pi e^{-\mu\left(\vartheta-p_{0}\right.}\right) & \sum_{n=1}^{\infty} \frac{1}{n !} \frac{1}{(n-1) !}\left(\frac{1}{2} \lambda \mu t\right)^{n} \\
& \times\left(\vartheta-p_{0}\right)^{n-1} \mathbf{1}_{\mathbb{R}_{+}}\left(\vartheta-p_{0}\right),
\end{aligned}
$$


we have that

$$
\begin{aligned}
\mathcal{F}^{-1}(g(\eta, t))(\vartheta)=\delta & \left(\vartheta-p_{0}\right) \\
+e^{-\mu\left(\vartheta-p_{0}\right)} & \sum_{n=1}^{\infty} \frac{1}{n !} \frac{1}{(n-1) !}\left(\frac{1}{2} \lambda \mu t\right)^{n} \\
& \times\left(\vartheta-p_{0}\right)^{n-1} \mathbf{1}_{\mathbb{R}_{+}}\left(\vartheta-p_{0}\right)
\end{aligned}
$$

and the assertion follows thereafter.

1b. Next with player A's sustainability threshold $M=2$ we end the game when the stock price drops twice. Under equation (4.8),

$$
\begin{aligned}
& e^{i \eta p_{0}} \theta \Phi^{*}(\theta, x, y) \\
&= {[[1-\gamma(x, \eta, \theta+y)][1-\gamma(x, 0, y)]]^{-1} } \\
& \times \frac{1}{\theta}[\gamma(x, 0, y)-\gamma(x, 0, \theta+y)][\gamma(1,0,0)-\gamma(x, 0, y)] \\
&+[1-\gamma(x, \eta, \theta+y)]^{-1} \frac{1}{\theta}[\gamma(1,0,0) \\
&-\gamma(x, 0, y)-\gamma(1,0, \theta)+\gamma(x, 0, \theta+y)] .
\end{aligned}
$$

Now

$$
\begin{aligned}
\gamma(0, \phi, \vartheta)=E u^{x_{1}} e^{-i \phi \pi_{1}} & \left.\right|_{u=0} E e^{-\Delta_{1} \vartheta} \\
& =E \mathbf{1}_{\left\{\pi_{1} \geq 0\right\}} e^{-i \phi \pi_{1}} \frac{\lambda}{\lambda+\vartheta} .
\end{aligned}
$$

With the pdf of $\pi_{1}$ defined in (4.2),

$$
\gamma(x, \eta, y)=E x^{X_{1}} e^{-i \eta \pi_{1}} e^{-y \Delta_{1}}
$$

due to position independent marking

$$
\begin{aligned}
\gamma(x, \eta, y) & =E x^{X_{1}} e^{-i \eta \pi_{1}} \frac{\lambda}{\lambda+y}\left[\mathbf{1}_{\left\{X_{1}=0\right\}}+\mathbf{1}_{\left\{X_{1}=1\right\}}\right] \\
& =\frac{\lambda}{\lambda+y}\left[\beta\left(0, \pi_{1}\right)+\beta\left(1, \pi_{1}\right)\right]
\end{aligned}
$$

where

$$
\begin{aligned}
& \beta\left(0, \pi_{1}\right)=E x^{X_{1}} e^{-i \eta \pi_{1}} \mathbf{1}_{\left\{X_{1}=0\right\}} \\
& =E e^{-i \eta \pi_{1}} \mathbf{1}_{\left\{\pi_{1} \geq 0\right\}}=\int_{s=0}^{\infty} \frac{1}{2} \mu e^{-(\mu+i \eta) s} d s=\frac{1}{2} \frac{\mu}{\mu+i \eta}
\end{aligned}
$$

and

$$
\begin{aligned}
\beta\left(1, \pi_{1}\right) & =E x^{X_{1}} e^{-i \eta \pi_{1}} \mathbf{1}_{\left\{X_{1}=1\right\}}=E x^{X_{1}} e^{-i \eta \pi_{1}} \mathbf{1}_{\left\{\pi_{1}<0\right\}} \\
& =x \int_{s=-\infty}^{0} e^{-i \eta s} \frac{1}{2} \mu e^{\mu s} d s=\frac{1}{2} \frac{\mu x}{\mu-i \eta}
\end{aligned}
$$

So

$$
\begin{aligned}
\gamma(x, \eta, y)= & \frac{\lambda}{\lambda+y}\left[\beta\left(0, \pi_{1}\right)+\beta\left(1, \pi_{1}\right)\right] \\
& =\frac{\lambda}{\lambda+y} \frac{1}{2} \frac{\mu^{2}(1+x)-i \eta \mu(1-x)}{\mu^{2}+\eta^{2}}
\end{aligned}
$$

and

$$
\gamma(x, 0, y)=\left.\gamma(x, \eta, y)\right|_{\eta=0}=\frac{\lambda}{\lambda+y} \frac{1+x}{2}
$$

with

$$
\gamma(1,0, y)=\frac{\lambda}{\lambda+y}
$$

Hence,

$$
\begin{aligned}
& e^{i \eta p_{0}} \theta \Phi^{*}(\theta, x, y) \\
= & {\left[\left[1-\frac{\lambda}{\lambda+\theta+y} \frac{1}{2} \frac{\mu^{2}(1+x)-i \eta \mu(1-x)}{\mu^{2}+\eta^{2}}\right]\right]^{-1} \frac{\theta}{\lambda+\theta} }
\end{aligned}
$$

implying that

$$
\begin{aligned}
& e^{i \eta p_{0}} \Phi^{*}(\theta, x, y) \\
= & e^{i \eta p_{0}} \int_{t=0}^{\infty} e^{-\theta t} D_{p} \mathcal{L} \mathcal{C}_{q}\left[E e^{-i \eta P(t)} \mathbf{1}_{\left[0, t_{\nu}\right)}(t)\right](x, y) d t \\
= & {\left[\left[1-\frac{\lambda}{\lambda+\theta+y} \frac{1}{2} \frac{\mu^{2}(1+x)-i \eta \mu(1-x)}{\mu^{2}+\eta^{2}}\right]\right]^{-1} \frac{1}{\lambda+\theta} . }
\end{aligned}
$$

Next, recalling (4.16) we have

$$
\begin{aligned}
{\left[\left[1-\frac{\lambda}{\lambda+\theta+y} \frac{1}{2}\right.\right.} & \left.\left.\frac{\mu^{2}(1+x)-i \eta \mu(1-x)}{\mu^{2}+\eta^{2}}\right]\right]^{-1} \\
& =\frac{1}{1-A \frac{1}{\lambda+\theta+y}}=1+A \frac{1}{\theta+y+B}
\end{aligned}
$$

and

$$
e^{i \eta p_{0}} \Phi^{*}(\theta, x, y)=\frac{1}{\lambda+\theta}+A \frac{1}{\lambda+\theta} \frac{1}{\theta+y+B} .
$$

The Laplace inverse with respect to $y$ and $\theta$ of

$$
F(y, \theta)=\frac{1}{\lambda+\theta} \frac{1}{y}+A \frac{1}{\lambda+\theta} \frac{1}{\theta+y+B} \frac{1}{y},
$$

because of

$$
\begin{aligned}
\mathcal{L}_{y}^{-1}[F(y, \theta)](T)= & \frac{1}{\lambda+\theta}+A \frac{1}{\lambda+\theta} \frac{1-e^{-(\theta+B) T}}{\theta+B} \\
= & \frac{1}{\lambda+\theta}+A \frac{1}{\lambda+\theta} \frac{1}{B+\theta} \\
& \quad-A \frac{1}{\lambda+\theta} \frac{1}{B+\theta} e^{-B T} e^{-\theta T}
\end{aligned}
$$

is

$$
\begin{aligned}
\mathcal{L}_{\theta}^{-1}\left\{\mathcal{L}_{y}^{-1}[F(y, \theta)](T)\right\}(t) & \\
= & \mathcal{L}_{\theta}^{-1}\left(\frac{1}{\lambda+\theta}\right)(t)+A \mathcal{L}_{\theta}^{-1}\left(\frac{1}{\lambda+\theta} \frac{1}{B+\theta}\right)(t) \\
& \quad-A e^{-B T} \mathcal{L}_{\theta}^{-1}\left(\frac{1}{\lambda+\theta} \frac{1}{B+\theta} e^{-\theta T}\right)(t) \\
= & e^{-\lambda t}+\frac{A}{B-\lambda} e^{-\lambda t}\left[1-\mathbf{1}_{(T, \infty)}(t) e^{(\lambda-B) T}\right] \\
& -\frac{A}{B-\lambda} e^{-B t}\left[1-\mathbf{1}_{(T, \infty)}(t)\right]
\end{aligned}
$$


So,

$$
\begin{aligned}
e^{i \eta p_{0}} \mathcal{L}_{\theta}^{-1} \mathcal{L}_{y}^{-1} \frac{1}{y} \Phi^{*}(\theta, 0, y)(t, T) & \\
=e^{-\lambda t}+\frac{A}{B-\lambda} e^{-\lambda t} & {\left[1-\mathbf{1}_{(T, \infty)}(t) e^{(\lambda-B) T}\right] } \\
& -\frac{A}{B-\lambda} e^{-B t} \mathbf{1}_{[0, T]}(t),
\end{aligned}
$$

where

$$
\begin{aligned}
& A=\frac{1}{2} \lambda \frac{\mu^{2}(1+x)-i \eta \mu(1-x)}{\mu^{2}+\eta^{2}} \\
& \quad \text { and } B=\lambda-A \text { and } \frac{A}{B-\lambda}=-1 .
\end{aligned}
$$

Hence

$$
\begin{gathered}
e^{i \eta p_{0}} \mathcal{L}_{\theta}^{-1} \mathcal{L}_{y}^{-1} \frac{1}{y} \Phi^{*}(\theta, 0, y)(t, T) \\
=e^{-\lambda t}-e^{-\lambda t}\left[1-\mathbf{1}_{(T, \infty)}(t) e^{A T}\right]+e^{-B t} \mathbf{1}_{[0, T]}(t) \\
=e^{-\lambda t}\left[e^{\frac{1}{2} \lambda t \frac{\mu}{\mu+i \eta}} e^{\frac{1}{2} \lambda t \frac{\mu x}{\mu-i \eta}} \mathbf{1}_{[0, T]}(t)\right. \\
\left.\quad+e^{\frac{1}{2} \lambda \frac{\mu}{\mu+i \eta} T} e^{\frac{1}{2} \lambda \frac{\mu x}{\mu-i \eta} T} \mathbf{1}_{(T, \infty)}(t)\right]
\end{gathered}
$$

Next we apply the $\mathcal{D}$-operator to

$$
\begin{aligned}
G(x)=e^{-\lambda t}\left[e^{\frac{1}{2} \lambda t \frac{\mu}{\mu+i \eta}} e^{\frac{1}{2} \lambda t \frac{\mu x}{\mu-i \eta}} \mathbf{1}_{[0, T]}(t)\right. \\
\\
\left.\quad+e^{\frac{1}{2} \lambda \frac{\mu}{\mu+i \eta} T} e^{\frac{1}{2} \lambda \frac{\mu x}{\mu-i \eta} T} \mathbf{1}_{(T, \infty)}(t)\right]
\end{aligned}
$$

to get

$$
\begin{gathered}
\mathcal{D}_{x}^{1} G(x) \\
=e^{-\lambda t}\left[e^{\frac{1}{2} \lambda t \frac{\mu}{\mu+i \eta}} \mathcal{D}_{x}^{1} \sum_{n=0}^{\infty} \frac{1}{n !}\left(\frac{1}{2} \lambda t \frac{\mu}{\mu-i \eta}\right)^{n} x^{n} \mathbf{1}_{[0, T]}(t)\right. \\
\left.+e^{\frac{1}{2} \lambda \frac{\mu}{\mu+i \eta} T} \mathcal{D}_{x}^{1} \sum_{n=0}^{\infty} \frac{1}{n !}\left(\frac{1}{2} \lambda T \frac{\mu}{\mu-i \eta}\right)^{n} x^{n} \mathbf{1}_{(T, \infty)}(t)\right] \\
=e^{-\lambda t}\left[e^{\frac{1}{2} \lambda t \frac{\mu}{\mu+i \eta}} \sum_{n=0}^{1} \frac{1}{n !}\left(\frac{1}{2} \lambda t \frac{\mu}{\mu-i \eta}\right)^{n} \mathbf{1}_{[0, T]}(t)\right. \\
\left.+e^{\frac{1}{2} \lambda \frac{\mu}{\mu+i \eta} T} \sum_{n=0}^{1} \frac{1}{n !}\left(\frac{1}{2} \lambda T \frac{\mu}{\mu-i \eta}\right)^{n} \mathbf{1}_{(T, \infty)}(t)\right] \\
=e^{-\lambda t}\left[e^{\frac{1}{2} \lambda t \frac{\mu}{\mu+i \eta}}\left[1+\frac{1}{2} \lambda t \frac{\mu}{\mu-i \eta}\right] \mathbf{1}_{[0, T]}(t)\right. \\
\left.\quad+e^{\frac{1}{2} \lambda \frac{\mu}{\mu+i \eta} T}\left[1+\frac{1}{2} \lambda T \frac{\mu}{\mu-i \eta}\right] \mathbf{1}_{(T, \infty)}(t)\right] .
\end{gathered}
$$

(4.54) implies that

$$
\begin{aligned}
& E e^{-i \eta P(t)} \mathbf{1}_{\left[0, t_{\nu}\right)}(t) \\
& =e^{-\left(\lambda t+i \eta p_{0}\right)}\left[e^{\frac{1}{2} \lambda t \frac{\mu}{\mu+i \eta}}\left[1+\frac{1}{2} \lambda t \frac{\mu}{\mu-i \eta}\right] \mathbf{1}_{[0, T]}(t)\right. \\
& \left.\quad+e^{\frac{1}{2} \lambda \frac{\mu}{\mu+i \eta} T}\left[1+\frac{1}{2} \lambda T \frac{\mu}{\mu-i \eta}\right] \mathbf{1}_{(T, \infty)}(t)\right] .
\end{aligned}
$$

In particular,

$$
\begin{aligned}
E \mathbf{1}_{\left[0, t_{\nu}\right)}(t) & =P\left\{t_{\nu}>t\right\} \\
= & e^{-\lambda t}\left[e^{\frac{1}{2} \lambda t}\left[1+\frac{1}{2} \lambda t\right] \mathbf{1}_{[0, T]}(t)\right. \\
& \left.\quad+e^{\frac{1}{2} \lambda T}\left[1+\frac{1}{2} \lambda T\right] \mathbf{1}_{(T, \infty)}(t)\right],
\end{aligned}
$$

or the $\mathrm{CDF}$ is

$$
\begin{aligned}
P\left\{t_{\nu} \leq t\right\}=1- & e^{-\lambda t}\left[e^{\frac{1}{2} \lambda t}\left[1+\frac{1}{2} \lambda t\right] \mathbf{1}_{[0, T]}(t)\right. \\
& \left.+e^{\frac{1}{2} \lambda T}\left[1+\frac{1}{2} \lambda T\right] \mathbf{1}_{(T, \infty)}(t)\right]
\end{aligned}
$$

The latter agrees with simulation as depicted in Figure 6 below. Again, we use the Monte Carlo approach to simulation for estimating $P\left\{t_{\nu} \leq t\right\}$ as described above.

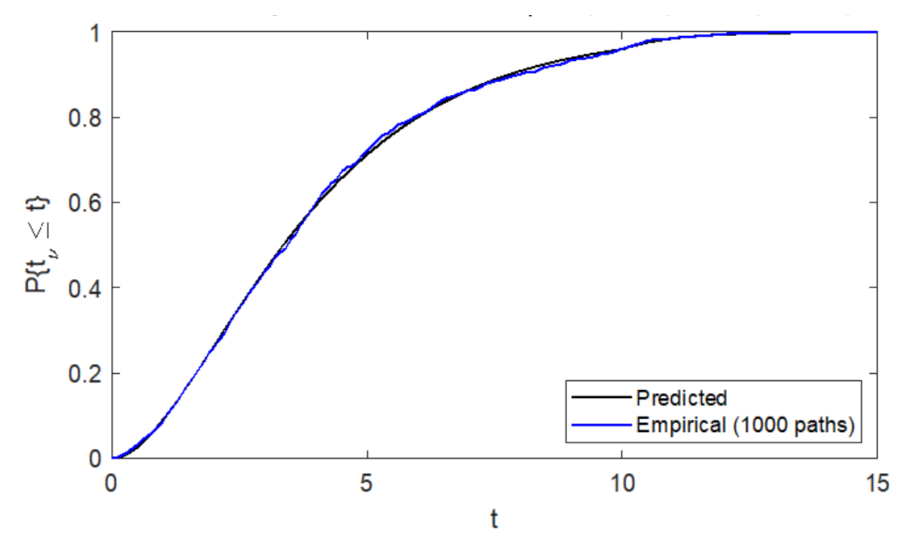

Figure 6. Predicted and empirical distribution functions $P\left(t_{\nu} \leq t\right)$ for a special case where $\mu=1, \lambda=1, T=10, p_{0}=4$, and $M=2$.

\section{Applications to Queueing Models}

In this section we return to queueing Models 2 and 3 regarding their relevance to the game studied in sections 3 and 4 . We restrict ourselves with a discussion on applications of these results moving more detailed and substantial treatments of these models in our separate, forthcoming, papers.

Model 2. Consider an GI/G/1-type queueing system in which a single server goes on vacations when the queue becomes exhausted. The input to the system is specified by a renewal process

$$
\mathcal{T}=\sum_{n=0}^{\infty} \varepsilon_{t_{n}}
$$

The server's (multiple) vacations consist of single vacation segments that form an ordinary Poisson process

$$
\mathcal{V}=\sum_{n=0}^{\infty} \varepsilon_{\tau_{n}}
$$

of rate $\lambda$ (vacation segments per unit time). The number of vacations was said to be "observed" or rather recorded only upon 
the epochs $\mathcal{T}$ making the combination of the two processes as a marked renewal process

$$
\mathcal{A} \otimes \mathcal{T}=\sum_{n=0}^{\infty} x_{n}
$$

with position dependent marking, where $x_{n}$ stands for the number of vacation segments in interval $\left[t_{n-1}, t_{n}\right)\left(t_{-1}=0\right)$. Thus,

$$
A_{n}=\sum_{k=0}^{n} x_{k}
$$

gives the number of vacation segments in interval $\left[0, t_{n}\right)$. As specified in the model, server's vacation segments may not exceed a positive integer number $M$. However, if he does, he will not be recalled until a next arrival epoch, say some $t_{k}$. Consequently, the real number of vacation segments the server renders can become arbitrarily larger than $M$ dependent on the crudeness of the point process $\mathcal{T}$. This happens because we do not have control over serve's vacations within intervals $\left[t_{n-1}, t_{n}\right)$.

On the other hand, the cumulative vacation time the server is allowed to use is also limited, namely, by a positive real number $T$. And again, once that number becomes exceeded, the server won't be recalled immediately, but with some delay upon a nearest arrival epoch. So the server will be recalled when one of the two named events take place, whichever of the two comes first.

In a nutshell, the server's vacations are terminated by the following formal means. With

$$
\begin{gathered}
\nu_{1}=\min \left\{n=0,1, \ldots: A_{n} \geq M\right\} \\
\nu_{2}=\min \left\{n=0,1, \ldots: t_{n} \geq T\right\} \\
\nu=\nu_{1} \wedge \nu_{2}
\end{gathered}
$$

we have $t_{\nu}$ being the server exit from his vacations (known in the literature as the first passage time). Now because the server vacations are being observed upon the arrival process $\mathcal{T}$, we guaranteed that the server finds at least one customer in the system to resume his service.

Note that the only reason to allow the arrival process to run from $t_{0}$ rather than $t_{1}$ is due some applications that could require a delay, but most practical reasons, the first observed time can be regarded as $t_{1}$. Thus, if the server is to return to the system at time $t_{1}$, he finds one customer waiting (or a batch of customers if the input is bulk).

As it readily seen, this model has no passive component that we can simply omit from the game. Alternatively, we can assume that there is yet another, independent, stream of customers which is a bulk Poisson formalized by a marked Poisson process

$$
\pi=\sum_{n=0}^{\infty} y_{n} \varepsilon_{s}
$$

with position independent marking, of intensity $\mu$. Let $\pi_{n}$ denote the number of customers that arrive in the system in inter$\operatorname{val}\left[t_{n-1}, t_{n}\right)$. Then,

$$
\mathcal{P}=\sum_{n=0}^{\infty} \pi_{n} \varepsilon_{t_{n}}
$$

is a marked renewal process describing the second input stream to the system as observed upon $\mathcal{T}$. Therefore, the marked random measure

$$
\mathcal{A} \otimes \mathcal{P} \otimes \mathcal{T}=\sum_{n=0}^{\infty}\left(x_{n}, \pi_{n}\right) \varepsilon_{t_{n}}
$$

is exactly as in the game-theoretic context of section 2, with the same notation and specifications. The main functional $\gamma$ of (2.3) is obviously

$\gamma(u, v, \vartheta)=E u^{x_{1}} v^{\pi_{1}} e^{-\vartheta \Delta_{1}}=\gamma(\vartheta+\lambda-\lambda u+\mu-\mu b(v))$,

where

$$
\gamma(\theta)=e^{-\theta \Delta_{1}}\left(\Delta_{1}=t_{1}-t_{0}\right)
$$

and

$$
b(v)=E v^{y_{1}} .
$$

(the size of the first batch in the second input).

When the sequence of vacations stopped and the server returns to the system, he may start servicing the available customers somehow separately or on the FIFO basis independent of their types. We are not going to worry about the next phase of a complex service cycle that initiates with vacations and ends with the completion of the first service thereafter, subject to a separate work. However, we need to know about the contents of the buffer (or waiting room) upon the end of the vacation and during the vacation for various reasons. One of them is to construct the semi-regenerative kernel of the queueing process in case of M/G/1-type or GI/M/1-type queues and a subsequent analysis of the continuous time parameter queueing process, cf., [1, 4-6]. Consequently, the time sensitive analysis rendered on phase 1 , during server's vacations and concluded in Theorem 3.1 will have to be merged with a time sensitive functional on phase 2, i.e. during a service time. The latter is relatively straightforward as the rest of the queue.

On a side note, if the queueing process is semi-regenerative, another useful approach would be based on the common formation of an embedded process while applying time insensitive analysis. The latter is far less complicated than Theorem 3.1 's scrutiny and it has been rendered in a series of work by the first author.

Model 3. In this M/G/1-type system, the server leaves the operating facility for maintenance, once the queue drops below some fixed $r(\geq 1)$. The term "maintenance" is almost synonymous to "vacations" except that the server can do some specified work, such as processing second priority customers until he is done with at least some $M$ of them or when his absence from the main facility exceeds $T$, whichever of the two events occurs first. Note that we assume that the server will processes second priority jobs in random batches and each service duration will correspond to a vacation segment and that runs by an independent renewal process $t_{0}, t_{1}, t_{2}, \ldots$. Now upon the server's return, he will not start his work on primary customers unless there are $N$ of them in the system, so that he is likely to rest waiting until the buffer accumulates to at least $N$ of them. The input is marked Poisson suggesting that his waiting time will be determined by using means of fluctuation analysis, in this case time sensitive analysis. Thus only upon the second 
exit does the server start servicing first priority customers in a batch not exceeding his capacity, say $R(r \leq R \leq N)$. So the service cycle in this version consists of three (not two) phases, namely, the first phase is the server maintenance, then his wait, and then service of primary customers.

The time insensitive analysis of comparable system has been undertaken by Dshalalow and Merie [18] and Dshalalow, Merie, and White [19]. The time sensitive analysis is, as expected significantly more complex, but we are done with the first phase by accommodating the results and notation of Theorem 3.1. In this case, the two active players A and B will be the secondary jobs during the maintenance period and the time spent during the maintenance, constrained by $M$ and $T$, respectively. The passive player $\mathrm{C}$ is impersonated by the stream of the primary jobs replenishing the buffer from the initial value $Q_{0}(<r)$ to whatever quantity it comes on server's return.

Here is another variant of the same model mentioned in the introduction. The server leaves the system under the same condition to render unspecified maintenance or he "leaves on vacations." The latter consists of identically distributed vacation segments formalized by a renewal point process $t_{1}, t_{2}, \ldots$ The server will continue his vacations until one of the two events takes places first. (a) The number of the primary units replenishes to at least $N$. (b) His absence exceeds time $T$. The ascribed rule is referred to the NT-policy and it is previously studied in the literature (already mentioned in the introduction). Now when the server returns, he may end up find less than $N$ jobs in the system. In this case, he rests waiting for the buffer to accumulate to $N$ or more customers before the server resumes his service. Like the above mentioned model, this one also consists of three phases. In the first phase, the input stream (presumably marked Poisson), that is player A, competes the vacation time, player B, bounded by $N$ and $T$, respectively. Obviously, one or both of these thresholds maybe exceeded, but maybe only one of them, so that the other player (the winner) will not see his site ruined upon the exit from the game. Note that in this model, there is no passive player, unless we want to invent one.

\section{Concluding Remarks}

In this paper we studied a class of signed marked random measures $\mathcal{A} \otimes \mathcal{P} \otimes \mathcal{T}=\sum_{n=0}^{\infty}\left(x_{n}, \pi_{n}\right) \varepsilon_{t_{n}}$ with position dependent marking, on a filtered probability space $\left(\Omega, \mathcal{F}, \mathcal{F}_{t}, P\right)$ that models a stochastic game of three players $\mathrm{A}, \mathrm{B}$, and $\mathrm{C}$, of whom $\mathrm{A}$ and $\mathrm{B}$ are active antagonistic players inflicting damages of random magnitudes upon each other at random epochs of time $\left\{t_{0}, t_{1}, \ldots\right\}$, whereas player $\mathrm{P}$, being possibly a collateral damage, does not respond to the attacks. The position of the passive player $\mathrm{C}$ is modeled by a non monotone marginal process $P(t)$ often related to stock price variations. We developed new techniques (in the framework of fluctuation theory), to find a closed-form expression for the functional

$$
\begin{aligned}
\Phi_{\nu}(t)=E z^{A(t)} e^{-i \eta P(t)} v^{A_{\nu-1}} e^{-i \varphi P_{\nu-1}} \\
\\
\times e^{-\psi t_{\nu-1}} u^{A_{\nu}} e^{-i \phi P_{\nu}} e^{-\vartheta t_{\nu}} \mathbf{1}_{\left[0, t_{\nu}\right)}(t)
\end{aligned}
$$

in terms of multiple transforms established in Theorem 3.1. With the inversion of the underlying transforms of $\Phi_{\nu}$, we provided ample evidence to the claim that $\Phi_{\nu}$ is analytically tractable, illustrating them in the analysis of stock option trading. The numerical values of analytical results agreed with simulation for a number of special cases.

We obtained analytical solutions for the functional

$$
\Phi_{\nu}(t)=E e^{-i \eta P(t)} \mathbf{1}_{\left[0, t_{\nu}\right)}(t) .
$$

This functional gives the status of underlying stock price process

$$
P(t)=\sum_{n=0}^{\infty} \pi_{n} \varepsilon_{t_{n}}[0, t]
$$

at any time in interval $\left[0, t_{\nu}\right)$. Specifically, using player's A threshold levels $M=1$ and $M=2$ which represent the first and second drop of the stock price process $P(t)$, respectively, we derive formulas for $E P(t) \mathbf{1}_{\left[0, t_{\nu}\right)}(t)$ and $P\left(t_{\nu}>t\right)$ and validated the analytical results by simulation. Furthermore, for $M=1$, we have establish an explicit formula for density $\frac{\partial}{\partial \vartheta} P\left\{P(t) \leq \vartheta, t_{\nu}>t\right\}$.

From the practical stand point, the setting went way beyond applications to stock option trading. We suggested several useful and popular queueing models that can be regarded as other examples of the game. Closed form results have been known in earlier work by the first author (c.f., [12-13]) and most recent [18-19], but they almost exclusively related to time insensitive analysis while time sensitive analysis associated with the continuous time parameter queueing process (meant to be in our context), still remains an open problem to date. So to the best of our knowledge, no previous work on this topic has been registered. As regards the queueing process, in the present paper, we laid foundation and obtained the most challenging results pertaining to phase 1 . This is when the server went on maintenance or vacations and ends phase 1 on his return. The other two phases are less challenging, but still require some work that will include merging of all subsequent phases to complete the queueing analysis. We note here that the entire work will involve a semi-regenerative analysis of the queueing process, which in turn will require the formation of the semiregenerative kernel. The latter deals with integrals of type

$$
h\left(Q_{0}, z\right)=\int_{t=0}^{\infty} E\left[z^{Q(t)} \mathbf{1}_{\left[0, T_{1}\right)}(t) Q_{0}\right] d t
$$

(cf. Dshalalow [15]) that is a marginal functional and reduction from

$$
\int_{t=0}^{\infty} e^{-\theta t} E\left[z^{Q(t)} \mathbf{1}_{\left[0, T_{1}\right)}(t) Q_{0}\right] d t
$$

when $\theta=0$ of Theorem 3.1.

\section{Acknowledgements}

The authors are indebted to anonymous referees whose insightful comments and suggestions contributed a great deal of improvement to clarity and presentation of our work. 


\section{REFERENCES}

[1] Abolnikov, L. and Dshalalow, J.H., Semi-regenerative analysis of controlled bulk queueing systems with a bilevel service delay discipline and some ergodic theorems, Computers Math. Appl., 25:3, 107-116, 1993.

[2] Alfa, A.S. and Frigui, I., Discrete NT-policy single server queue with Markovian arrival process and phase type service, European Journal of Operations Research, 88:3 (1996), 599-613.

[3] Alfa, A.S. and Li, W., Optimal policies for M/M/m queue with two different kinds of (N,T)-policies, Nav. Res. Logistics, 47:3 (2000), 240-258.

[4] Al-Matar, N. and Dshalalow, J.H., Maintenance in single-server queues. A game-theoretic approach, Math. Probl. in Eng., Vol. 2009, 23 pp.

[5] Al-Matar, N. and Dshalalow, J.H., A game-theoretic approach in single-server queues with maintenance. Time sensitive analysis, Commun. in Appl. Nonlin. Analysis, 17, No. 1 (2010), 65-92.

[6] Al-Matar, N. and Dshalalow, J.H., Time sensitive functionals in classes of queues with sequential maintenance, Stochastic Models, 27 (2011), 687-704.

[7] Bingham, N.H., Random walk and fluctuation theory, in Handbook of Statistics (Eds. D.N. Shanbhag and C.R. Rao), 19 (2001), Elsevier Science, 171-213.

[8] Borokov, A.A., On the first passage time for one class of processes with independent increments. Theor. Probab. Appl., 10 (1964), 331-334.

[9] Coutin, L. and Dorobantu, D., First passage time law for some Levy processes with compound Poisson: Existence of a density, Bernoulli, 17:4 (2011), 1127-1135.

[10] Dshalalow, J.H., Queueing systems with state dependent parameters, in: Frontiers in Queueing, Dshalalow, ed., CRC Press, 61-116, 1997.

[11] Dshalalow, J.H., Queues with hysteretic control by vacation and post-vacation periods. Queueing Systems, 29, 231-268, 1998.

[12] Dshalalow, J.H., Fluctuations of recurrent processes and their application to the stock market, Stochastic Analysis and Applications, 22:1, 2004 (67-79).

[13] Dshalalow, J.H., On exit times of a multivariate random walk with some applications to finance, Nonlinear Analysis, 63 (2005), 569-577.

[14] Dshalalow, J.H., Random walk analysis in antagonistic stochastic games, Stoch. Anal. and Appl., 26 (2008), 738-783.

[15] Dshalalow, J.H., Stochastic Processes, Lecture Notes, FIT, Melbourne, FL, 2015.

[16] Dshalalow, J.H. and Liew, A., On fluctuations of a multivariate random walk with some applications to stock options trading and hedging, Math. and Comp. Modeling, 44:10 (2006), 931944.

[17] Dshalalow, J.H. and Nandyose, K., Continuous time interpolation of monotone marked random measures and their applications, Neural, Parallel, and Sci. Comp., 26 (2018), 119-141.
[18] Dshalalow, J.H. and Merie, A., Fluctuation analysis in queues with several operational modes and priority customers, TOP, 26 (2018), 309-333.

[19] Dshalalow, J.H., Merie, A., and White, R.T., Fluctuation analysis in parallel queues with hysteretic control. Methodology and Computing in Applied Probability. Appeared online at doi.org/10.1007/s11009-019-09701-z.

[20] Dshalalow, J.H. and Nandyose, K., Real time analysis of signed marked random measures with applications to finance and insurance, Nonlinear Dynamics and System Theory, 19:1 (2019), $36-54$.

[21] Dshalalow, J.H. and White, R.T., Time sensitive analysis of independent and stationary increment processes, Journ. of Math. Anal. and Appl., 443 (2016), 817-833.

[22] Hellmund, G., Completely random signed measures, Statisitcs and Probability Letters, 79 (2009), 894-898.

[23] Heyman, D.P., The T-Policy for the M/G/1 queue, Manag. Science, 23:7 (1977), 775-778.

[24] Hida, T. (Editor), Mathematical Approach to Fluctuations: Astronomy, Biology and Quantum Dynamics: Proceedings of the Iias Workshop: Kyoto, Japan, May 18-21, 1992, World Scientific Publishers, 1995.

[25] Hur, S., Kim, J.S., and Kang, C.W., An analysis of the M/G/1 system with $N$ and $T$ policy, Applied Math. Modelling, 27:8 (2003), 665-675.

[26] Kadankov, V.F. and Kadankova, T.V., On the distribution of the moment of the first exit time from an interval and value of overjump through borders interval for the processes with independent increments and random walk, Random Operators and Stochastic Equations, 13:3 (2005), 219-244.

[27] Kuo, C-C, Ke, J-C, and Choudhury, G., Optimal NT policies for a two-phase service M/G/1 system with Bernoulli vacation schedule, Quality Technology \& Quantitative Management, 12:3 (2015), 343-353.

[28] Kyprianou, A.E. and Pistorius, M.R., Perpetual options and Canadization through fluctuation theory, Ann. Appl. Prob., 13:3 (2003), 1077-1098.

[29] Lee, H.W., Seo, W.J., and Lee, S.W., Analysis of the M/G/1 queueing system under the triadic (N, D, T)-Policies, Quality Technology and Qualitative Management, 8:3 (2011), 333-357.

[30] Mellander, E., Vredin, A, and Warne, A., Stochastic trends and economic fluctuations in a small open economy, J. Applied Econom., 7:4 (1992), 369-94.

[31] Muzy, J., Delour1, J., and Bacry, E., Modelling fluctuations of financial time series: from cascade process to stochastic volatility model, Eur. Phys. J., B 17 (2000), 537-548.

[32] Redner, S., A Guide to First-Passage Processes, Cambridge University Press, Cambridge, 2001.

[33] Shinozuka, M. and Wu, W-F., On the first passage problem and its application to earthquake engineering, Proceedings of Ninth World Conference on Earthquake Engineering, August 29, (VIII) 1988, Tokyo-Kyoto, Japan. 
[34] Smorodina, N. and Faddeev M., The Lévy-Khinchin representation of the one class of signed stable measures and some of its applications, Acta Appl. Math., 110 (2010), 1289-1308.

[35] Tadj, L., On an M/G/1 quorum queueing system under T-policy, JORS, 54:5 (2003), 466-471.

[36] Takács, L., On fluctuations of sums of random variables, in Studies in Probability and Ergodic Theory. Advances in Mathematics; Supplementary Studies, Volume 2, ed. by G.-C. Rota, (1978), 45-93.

[37] Wang, T-Y, Wang, K-H, and Pearn, W.L., Optimization of the T policy M/G/1 queue with server breakdowns and general startup times, Journ. Appl. and Comput. Math., 228 (2009), 270-278.
[38] Yin, C., Wen, Y., Zong, Z., and Shen, Y., The first passage time problem for mixed-exponential jump processes with applications in insurance and finance, Abstract and Applied Analysis (2014), 9 pages.

[39] Yu, M. and Alfa, A.S., Some analysis results associated with the optimization problem for a discrete-time finite-buffer NT-policy queue, Operational Res., 16 (2016), 161-179.

[40] Zhang, X., Wanga, J., Doc, T.V., Threshold properties of the M/M/1 queue under T-policy with applications, Applied Mathematics and Computation, 262:15 (2015), 284-301. 